Penning transfer in argon-based gas mixtures

This article has been downloaded from IOPscience. Please scroll down to see the full text article. 2010 JINST 5 P05002

(http://iopscience.iop.org/1748-0221/5/05/P05002)

View the table of contents for this issue, or go to the journal homepage for more

Download details:

IP Address: 137.138.124.142

The article was downloaded on 11/07/2011 at 09:22

Please note that terms and conditions apply. 


\title{
Penning transfer in argon-based gas mixtures
}

\author{
Ö. Şahin, ${ }^{a, 1}$ i. Tapan, ${ }^{a}$ E.N. Özmutlu ${ }^{a}$ and R. Veenhof ${ }^{b, c}$ \\ ${ }^{a}$ Department of Physics, Uluda $\breve{g}$ University, \\ 16059 Bursa, Turkey \\ ${ }^{b}$ Physics Department, Room 2320 Chamberlin Hall, University of Wisconsin-Madison, \\ 1150 University Avenue, Madison, WI 53706-1390, U.S.A. \\ ${ }^{c}$ CERN, PH department, \\ CH-1211 Gen'eve 23, Switzerland
}

E-mail: osahin@uludag.edu.tr

ABSTRACT: Penning transfers, a group of processes by which excitation energy is used to ionise the gas, increase the gas gain in some detectors. Both the probability that such transfers occur and the mechanism by which the transfer takes place, vary with the gas composition and pressure. With a view to developing a microscopic electron transport model that takes Penning transfers into account, we use this dependence to identify the transfer mechanisms at play. We do this for a number of argon-based gas mixtures, using gain curves from the literature.

KEYWORDS: Gaseous detectors; Ionization and excitation processes; Charge transport and multiplication in gas; Detector modelling and simulations II (electric fields, charge transport, multiplication and induction, pulse formation, electron emission, etc)

\footnotetext{
${ }^{1}$ Corresponding author
} 


\section{Contents}

1 Ionisations through excitation $\quad 1$

1.1 Penning effect 1

1.2 Excitation dissipation mechanisms 2

1.3 Transfer probability model 4

2 Properties of the gas mixtures 6

2.1 Collision frequency 6

2.2 Argon spectroscopy

2.3 Xenon spectroscopy 9

2.4 Argon excimers 9

$\begin{array}{lll}2.5 & \text { Associative ionisation } & 10\end{array}$

$\begin{array}{ll}2.6 \text { UV absorption } & 10\end{array}$

3 Transfer probability measurement $\quad 11$

3.1 Townsend coefficient adjustment 11

3.2 Gain calibration 11

3.3 Photon feedback 12

$\begin{array}{lll}3.4 & \text { Other effects } & 12\end{array}$

4 Experimental data $\quad 13$

4.1 Argon-xenon mixtures 13

$\begin{array}{ll}4.2 & \text { Argon-carbon dioxide mixtures } \\ & 16\end{array}$

$\begin{array}{ll}4.3 & \text { Argon-ethyne mixtures } \\ & 17\end{array}$

4.4 Argon-methane mixtures 19

4.5 Argon-ethane, argon-propane and argon-isobutane mixtures 23

5 Conclusion $\quad 25$

\section{Ionisations through excitation}

\subsection{Penning effect}

Electron multiplication in gas-based detectors sometimes far exceeds the gain calculated using Townsend coefficients alone. This is most notably the case if a gas with a low ionisation potential is added to a gas with higher-energy excited states.

The additional gain is accounted for by the transformation of excitation energy into ionisations. Although the ionisation cross section is often higher than the excitation cross section, excitations are abundantly produced because of their lower energy threshold (figure 1). The gain enhancement is named "Penning effect" after Frans Michel Penning who worked from 1924 at the Philips 

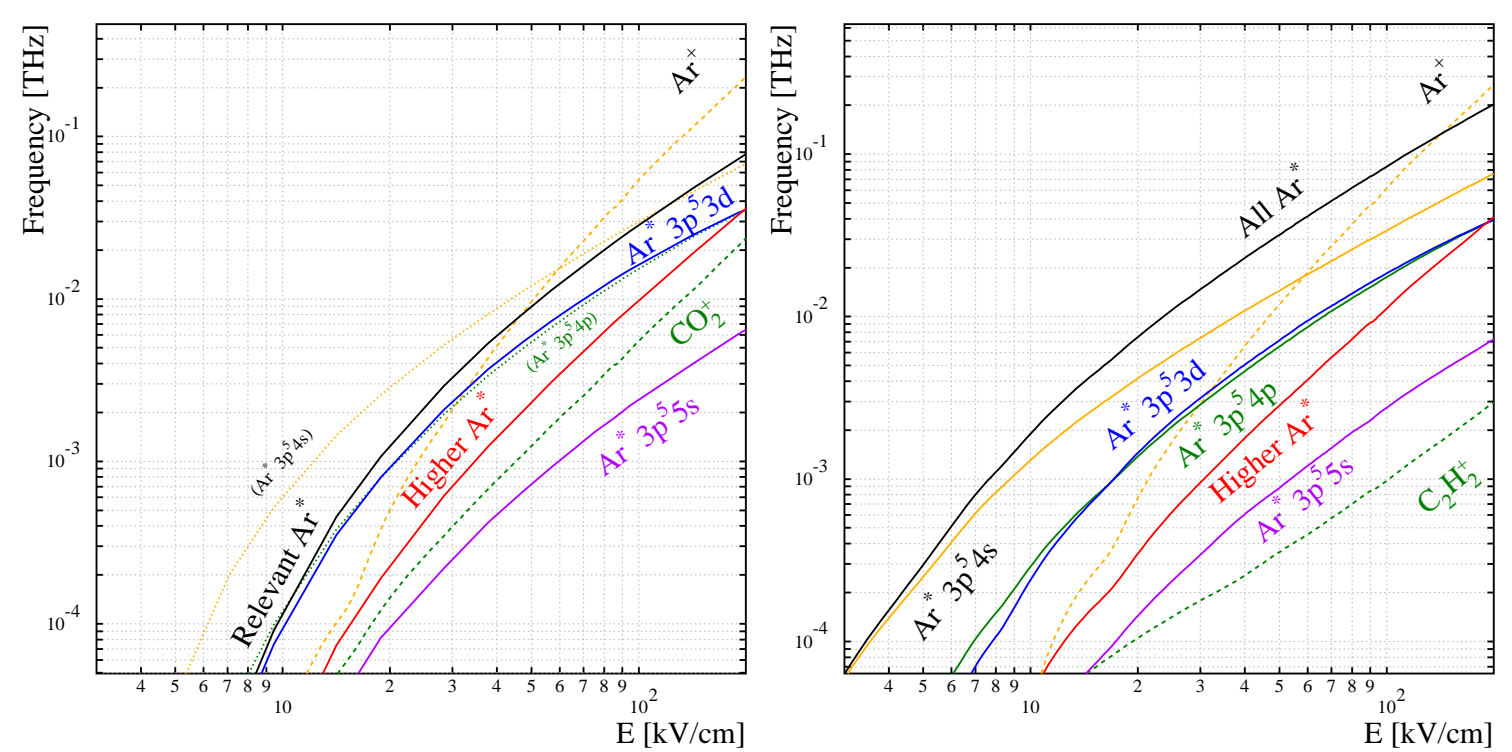

Figure 1. Left: Excitation (solid lines) and ionisation frequencies (dashed lines) in $\mathrm{Ar} 90 \% \mathrm{CO}_{2} \quad 10 \%$, which has a moderate Penning effect (left), and $\mathrm{Ar} 99.5 \% \mathrm{C}_{2} \mathrm{H}_{2} 0.5 \%$, which has one of the strongest known Penning effects (right). Intransferable excitations are dotted and are not included in the total excitation frequency. These mixtures are discussed in sections 4.2 and 4.3. Calculated using Magboltz 8.6 [14].

research laboratories (Eindhoven) on gas discharges, and noted that the discharge potential in mixtures of neon, argon and mercury is lower than in pure noble gases $[74,75]$. He attributed the effect to metastables. For a detailed account, see [30].

Penning transfers have significant repercussions: the Jesse effect, the reduced work function in Penning mixtures [46], is the result of energy transfers and the Penning effect also modifies the Fano factor in such mixtures [7, 59].

In the following, we first develop a model for the concentration and pressure dependence of ionising energy transfer. Comparing the model with transfer probabilities extracted from experimental data, we try and identify the mechanisms that are at play in the various gas mixtures. We consider only binary mixtures of a noble gas $A$ (argon) and a quenching admixture $B$. The admixture can be an organic gas, $\mathrm{CO}_{2}$ or another noble gas. Transfer probabilities have been measured before $[9,13]$ but a study with a view to producing a microscopic model of the transfers, is in as far as we know still lacking.

\subsection{Excitation dissipation mechanisms}

Excited noble gas atoms, as opposed to noble gas atoms in their ground state, are highly reactive. On account of their single electron in an outer shell, they have chemical properties similar to those of the alkali metals. They also have the low ionisation potential typical of alkali metals [81]. Thus, in most cases, it is excited noble gas that ionises the admixture.

Penning transfer is a balance between a range of transfer reactions and non-ionising decay of excited states. The most common transfer mechanism is the two-body collision of an excited atom 
$A^{*}$ with an admixture molecule $B$ resulting in the ionisation of $B$ :

$$
\begin{aligned}
& A^{*}+B \rightarrow(A B)^{+}+e^{-} \\
& A^{*}+B \rightarrow A+B^{+}+e^{-}
\end{aligned}
$$

Two processes have been proposed for the details of the ionisation. In the exchange reaction, an electron tunnels from $B$ into $A^{*}$ to fill the hole, after which an excited electron is ejected from $A^{*}$ :

$$
\begin{aligned}
A^{*}\left(e_{A}^{-}\right)+B\left(e_{B}^{-}\right) \rightarrow & A^{*-}\left(e_{A}^{-} e_{B}^{-}\right)+B^{+} \\
& A^{*-}\left(e_{A}^{-} e_{B}^{-}\right) \rightarrow A\left(e_{B}^{-}\right)+e_{A}^{-}
\end{aligned}
$$

This mechanism, akin to Auger emission, can occur when radiative decay is quantum-mechanically forbidden. It was proposed by H. Hotop and A. Niehaus [41] to explain the similarity of transfer rates for excited states with vastly different radiative lifetimes. The process requires close proximity of the excited and non-excited molecules.

Direct transfer consists of a pair of optical transitions: $A^{*}$ returns to its ground state while an electron from $B$ is ejected [49]:

$$
\begin{aligned}
A^{*}\left(e_{A}^{-}\right) & \rightarrow A\left(e_{A}^{-}\right) \\
B\left(e_{B}^{-}\right) & \rightarrow B^{+}+e_{B}^{-}
\end{aligned}
$$

This process works over distances that are large compared with the molecular dimensions, but owing to the $1 / r^{3}$ dependence of dipole-dipole couplings, it more easily occurs between nearby molecules [83].

One speaks of (heteronuclear) associative ionisation if $A^{*}$ and $B$ bind to form a molecular ion. In Hornbeck-Molnar ionisation, or homonuclear associative ionisation (h.a.i.), $A$ and $B$ are of the same species. Both types of associative ionisation occur in particular with highly excited noble gas atoms: the reaction thresholds are noticeably lower than the individual ionisation potentials (section 2.5). Homonuclear associative ionisation is understood to be a two-step process: first an excited atom is formed which later binds with a ground state atom. Measurements are consistent with each stage being proportional with pressure [40], which suggests that the binding step which concerns us, is a two-body process. Although it was originally believed that only metastable $A^{*}$ contribute, the process is in fact dominated by higher, non-metastable excitations.

A bound state of an excited noble gas atom and one in the ground state is known as "excimer". Initially formed in a two- or three-body collision, the quantum state can be changed by further collisions. In argon at atmospheric pressure, the three-body nature of the process is accepted. See e.g. [90] for a discussion of the two-body contribution. Excimers decay under VUV emission, which can in general not ionise. They can also excite and ionise certain admixture molecules:

$$
\begin{aligned}
A^{*}+2 A \rightarrow & A_{2}^{*}+A \\
& A_{2}^{*}+B \rightarrow 2 A+B^{*} \\
& A_{2}^{*}+B \rightarrow 2 A+B^{+}+e^{-}
\end{aligned}
$$

Energy transfer can further occur following the radiative decay of excited states. Photons with an energy above the ionisation potential of the admixture have a mean free path measured in 

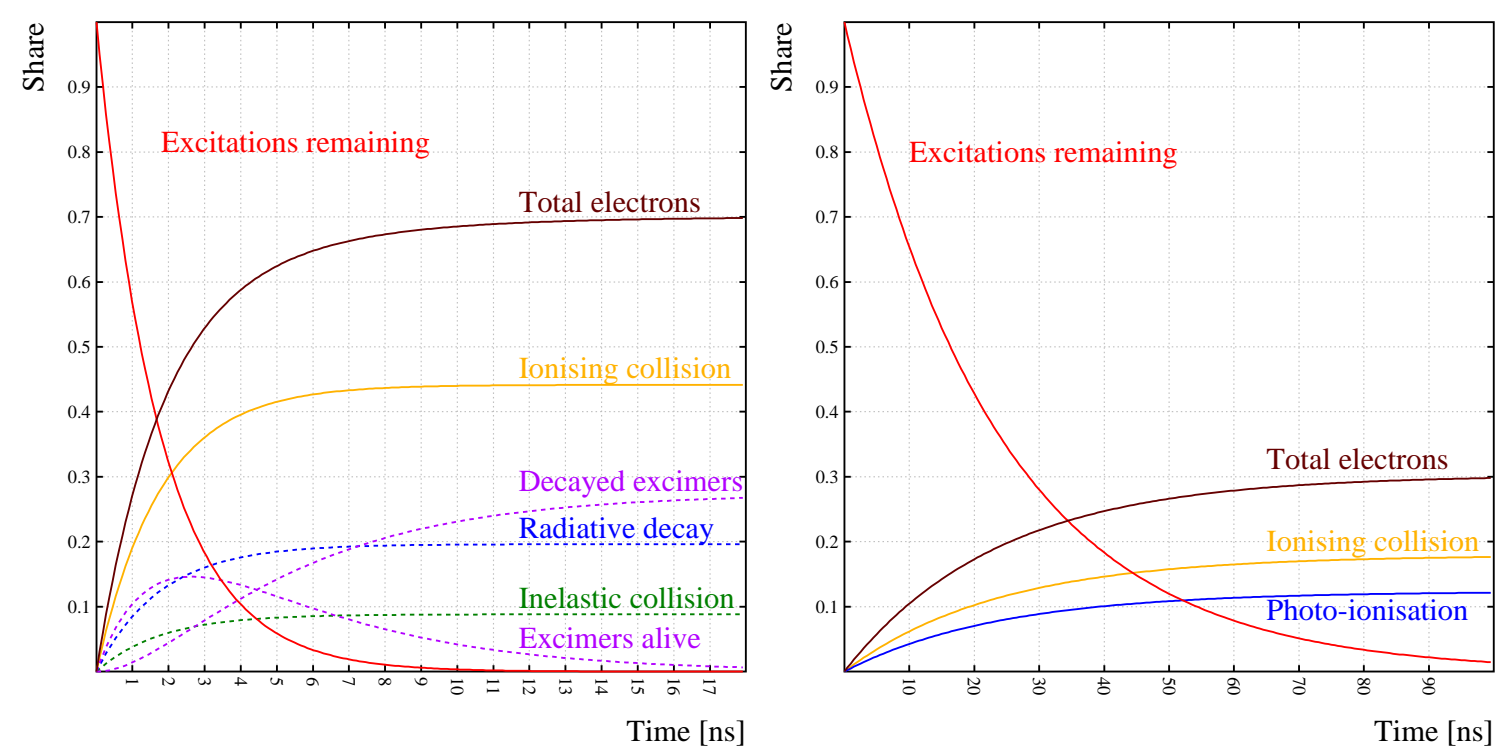

Figure 2. Left: Dissipation of excited states in a hypothetical gas with $10 \%$ admixture through ionising collisions $\left(f_{B^{+}}=0.5, \tau_{A^{*} B}=2 \mathrm{~ns}, f_{A^{+}}=0\right.$, ), inelastic collisions $\left(f_{\bar{B}}=0.1, f_{\bar{A}}=0\right)$, radiative decay $\left(\tau_{A^{*}}=\right.$ $\left.9 \mathrm{~ns}, f_{\mathrm{rad}}=0.9\right)$ and excimers $\left(f_{A_{2}}=0.1, \tau_{A^{*} A}=0.64 \mathrm{~ns}, \tau_{A_{2}}=4.2 \mathrm{~ns}, f_{\mathrm{exc}}=0.3\right)$. Shown are the cumulative numbers of collisions, radiative decays and decayed excimers, and the residual numbers of excimers and excited states. The number of electrons is the cumulative sum of collision transfers, photoelectric effect and excimer-related ionisations. Photo-ionisation and excimer-induced ionisation are not shown. Right: Dissipation in $\mathrm{Ar} 90 \% \mathrm{CH}_{4} 10 \%$ at $p=12$ atm calculated using parameters extracted from gain curves (section 4.4), a mean lifetime $\tau_{A^{*}}=40 \mathrm{~ns}$ (section 2.2) and the collision times $\tau_{A^{*} A}$ and $\tau_{A^{*} B}$ (table 1).

$\mu \mathrm{m}$ (section 2.6) and ionise the gas in or near the avalanche via the photoelectric effect. Photoionisation tends to be suppressed if the decay proceeds in two or more steps. This is e.g. the case for the $3 p^{5} 4 p$ argon levels (section 2.2) for which radiative decay directly to the ground state is forbidden. Low-energy photons have a substantial range and can generate photo-electrons on metal cathodes provided the energy is larger than the work function. This can lead to secondary avalanches (section 3.3).

Energy can be dissipated through inelastic collisions that excite the vibration, rotation and polyad modes of the admixture. Noble gases do not have inelastic cross section terms, but xenon readily picks up excess energy from excited argon atoms (section 4.1).

\subsection{Transfer probability model}

Some energy transfer and energy loss mechanisms are, as shown above, dominated by three-body interactions, others rely on two-body collisions or at least on close proximity, and still others are decay processes independent of the environment. Such differences in mechanism translate to differences in scaling with partial pressure, and we will use the latter to study the transfer process.

To construct a model for the partial pressure dependence of the transfer probability, we follow an excited atom $A^{*}$ through a series of small time steps of duration $\mathrm{d} t$. At each step, the excited $A^{*}$ atom can lose its energy through the following processes (the notation is summarised at the end of this section): 
- $p c f_{B^{+}} \mathrm{d} t / \tau_{A^{*} B}$ : collision with ionising energy transfer $\left(A^{*}+B \rightarrow A+B^{+}+e^{-}\right)$;

- $p(1-c) f_{A^{+}} \mathrm{d} t / \tau_{A^{*} A}$ : homonuclear associative ionisation $\left(A^{*}+A \rightarrow A_{2}^{+}+e^{-}\right)$;

- $p^{2}(1-c)^{2} f_{A_{2}} \mathrm{~d} t / \tau_{A A A}$ : excimer formation $\left(A^{*}+2 A \rightarrow A_{2}^{*}+A\right)$;

- $p c f_{\bar{B}} \mathrm{~d} t / \tau_{A^{*} B}$ : loss of excitation energy by collision with a $B$ molecule;

- $p(1-c) f_{\bar{A}} \mathrm{~d} t / \tau_{A^{*} A}$ : loss of excitation energy by collision with an $A$ molecule;

- $\mathrm{d} t / \tau_{A^{*}}$ : decay, e.g. radiative $\left(A^{*} \rightarrow A+\gamma\right)$.

Ionising collisions, including homonuclear associative ionisation, produce ionisation electrons directly while radiative decay photons and excimers can do so in a second stage. The probabilities $f_{\text {rad }}$ (discussed below) respectively $f_{\text {exc }}$ for these processes depend on the pressure $p$, on the mixing proportion $c$ and on photo cross sections. The number of ionisation electrons resulting from this step can therefore be writted as $k \mathrm{~d} t$ where

$$
k=p c \frac{f_{B^{+}}}{\tau_{A^{*} B}}+p(1-c) \frac{f_{A^{+}}}{\tau_{A^{*} A}}+p^{2}(1-c)^{2} \frac{f_{A_{2}} f_{\mathrm{exc}}}{\tau_{A A A}}+\frac{f_{\mathrm{rad}}}{\tau_{A^{*}}}
$$

The probability that the excited state survives the step, and thus remains available for the production of electrons in the next step, is $1-\mathrm{d} t / \tau_{P}$, where $\tau_{P}$ is given by

$$
\frac{1}{\tau_{P}}=p c \frac{f_{B^{+}}+f_{\bar{B}}}{\tau_{A^{*} B}}+p(1-c) \frac{f_{A^{+}}+f_{\bar{A}}}{\tau_{A^{*} A}}+p^{2}(1-c)^{2} \frac{f_{A_{2}}}{\tau_{A A A}}+\frac{1}{\tau_{A^{*}}}
$$

Summing the number of electrons produced in successive time steps, under the condition that the excited atom has survived all previous steps, the total number of electrons produced by an excited atom turns out to be:

$$
\begin{aligned}
r(p, c) & =k \mathrm{~d} t+k \mathrm{~d} t\left(1-\mathrm{d} t / \tau_{P}\right)+k \mathrm{~d} t\left(1-\mathrm{d} t / \tau_{P}\right)^{2}+\cdots \\
& =k \tau_{P}
\end{aligned}
$$

Assuming that decay photons do not reach the cathode, the probability that $A^{*}$ radiates a photon which ionises, is formally given by:

$$
f_{\mathrm{rad}}=f_{\gamma} \frac{c \sigma_{\mathrm{pi}}^{B}(\gamma)+(1-c) \sigma_{\mathrm{pi}}^{A}(\gamma)}{c \sigma_{\mathrm{pa}}^{B}(\gamma)+(1-c) \sigma_{\mathrm{pa}}^{A}(\gamma)}
$$

In practice, we should set $\sigma_{\mathrm{pi}}^{A}=\sigma_{\mathrm{pa}}^{A}=0$ because absorption of the photon by an $A$ atom merely creates, at some distance from the original $A^{*}$, a new $A^{*}$ which starts its own dissipation cycle. As a result, $f_{\text {rad }}$ is independent of $c$ and proportional to the photo-ionisation yield $\eta^{B}$ :

$$
f_{\mathrm{rad}}=f_{\gamma} \sigma_{\mathrm{pi}}^{B}(\gamma) / \sigma_{\mathrm{pa}}^{B}(\gamma)=f_{\gamma} \eta^{B}(\gamma)
$$

Similarly, $f_{\text {rad }}$ is independent of pressure: pressure modifies the mean free path of photons, but not the photo-ionisation cross section. The expression ceases to be valid when $c \rightarrow 0$ where $f_{\text {rad }} \rightarrow 0$ for lack of $B$ molecules on the way to the cathode. The range of applicability can be estimated 
from $\exp \left(-d N c \sigma_{\mathrm{pa}}^{B}\right) \ll 1$ and is e.g. $c \gg 0.3 \%$ for a distance $d=0.5 \mathrm{~cm}$ to the cathode and a photo-absorption cross section of $25 \mathrm{Mb}$.

The life cycle of excimers resembles that of the excited states and is not discussed in detail.

The time dependence of the process is illustrated in figure 2. One notes the exponential dissipation of excited states with a time constant $\tau_{P}$. Electrons emerge with the same time constant through $A^{*}-B$ transfer collisions and through the photoelectric effect. Electron production via excimers, a two-step process, starts later and has a non-exponential time-dependence.

Notation: $c(0 \leq c<1)$ : number fraction of admixture molecules $B$ present in the mixture; $p$ : dimensionless pressure related to the gas pressure $p_{\text {gas }}$ by $p_{\text {gas }}=p \times 1 \mathrm{~atm} ; T_{\text {gas }}$ : temperature of the gas in $\mathrm{K} ; N=p_{\text {gas }} / k_{\mathrm{B}} T_{\text {gas }}$ : number of gas molecules per unit volume; $\tau_{A^{*} A}, \tau_{A^{*} B}, \tau_{A A A}$ etc.: mean time between $A^{*}-A, A^{*}-B, A^{*}-A-A$ collisions; $f_{\bar{A}}, f_{\bar{B}}$ : probability that $A^{*}$ loses its energy in a collision with $A$ or $B ; f_{A^{+}}, f_{B^{+}}, f_{A^{+}}^{\text {exc }}, f_{B^{+}}^{\text {exc }}$ : probability that $A$ or $B$ is ionised by colliding with $A^{*}$ or $A_{2}^{*}$; $f_{A_{2}}$ : probability that an $A^{*}-A$ collision results in excimer formation; $f_{\text {exc }}$ : probability that an excimer eventually produces an electron contributing to the avalanche; $\sigma_{\mathrm{pa}}^{A}(\gamma), \sigma_{\mathrm{pa}}^{B}(\gamma), \sigma_{\mathrm{pi}}^{A}(\gamma), \sigma_{\mathrm{pi}}^{B}(\gamma)$ : photo-absorption (photo-ionisation included) and photo-ionisation cross sections; $\tau_{A^{*}}, \tau_{A_{2}^{*}}$ : lifetime of the excited state and of an excimer; $f_{\gamma}$ : probability that a state undergoes radiative decay; $f_{\text {rad}}$ : $f_{\gamma}$ multiplied with the probability that the photon subsequently ionises a $B$ molecule, producing an electron which contributes to the avalanche, see eq. (1.14).

\section{Properties of the gas mixtures}

\subsection{Collision frequency}

The relation between partial pressure and collision frequency can be calculated starting from the mean $\bar{v}$ of the Maxwell velocity distribution for gas molecules of mass $m$ :

$$
\bar{v}=\sqrt{\frac{8 k_{\mathrm{B}} T_{\mathrm{gas}}}{\pi m}}
$$

At room temperature, argon atoms e.g. have a mean velocity of $400 \mathrm{~m} / \mathrm{s}$. The mean free path $\lambda$ is determined by the diameter $d$ of the molecules involved:

$$
\lambda=\frac{1}{\sqrt{2} N \pi d^{2}}
$$

The factor $\sqrt{2}$ corrects for the mean relative velocity being larger than the mean velocity by that factor. Combining the mean free path and the velocity, the mean time between collisions is:

$$
\tau=\frac{\sqrt{m k_{\mathrm{B}} T_{\mathrm{gas}}}}{4 \pi d^{2} p_{\mathrm{gas}}}
$$

For heterogeneous mixtures, we substitute the partial pressure of the admixture for $p_{\text {gas }}$, the mean diameter $\left(d_{1}+d_{2}\right) / 2$ for $d$ and the reduced mass $1 / m_{1}+1 / m_{2}$ for $2 / m$.

Collision times $\tau$ relevant for this paper, calculated using eq. (2.3), are listed in table 1 . The diameters are the most problematic ingredient. Numerous definitions are in use, each has its domain of applicability. For associative ionisation we take the covalent diameter, i.e. the length a covalent 
Table 1. Covalent diameters ${ }^{c}$ [93], diameters calculated from bond lengths and angles ${ }^{b}$ [12, 20, 44, 45], Dirac-Fock outer orbital maxima $^{m}$ [28], kinetic diameters ${ }^{k}$ [6, 25, 34, 42, 73, 84, 96], Deutsch-Märk diameters of excited Ar [29], Hartree-Fock outer orbital maxima of excited Xe [60], molecular weights and mean time $\tau_{A^{*} B}$ until an excited $A^{*}$ meets a $B$ molecule. Values for atmospheric pressure and $c=1$. Not all publications cited are primary references.

\begin{tabular}{|l|r|r|r|r|}
\hline Collision & $\begin{array}{r}\text { Diameter } A^{*} \\
{[\mathrm{pm}]}\end{array}$ & $\begin{array}{r}\text { Diameter } B \\
{[\mathrm{pm}]}\end{array}$ & $\begin{array}{r}\text { Mass } B \\
{[\mathrm{~g} / \mathrm{mol}]}\end{array}$ & $\begin{array}{r}\tau_{A^{*} B} \\
{[\mathrm{ps}]}\end{array}$ \\
\hline $\mathrm{Ar}{ }^{*}-\mathrm{Ar}$ & $5 p: 1630$ & $138^{m}, 194-212^{c}, 340^{k}$ & 39.948 & 28 \\
$\mathrm{Ar}-\mathrm{Xe}$ & $4 p: 680,3 d: 872$ & $206^{m}, 260-280^{c}, 396^{k}$ & 131.293 & 130,88 \\
$\mathrm{Ar}^{*}-\mathrm{CO}_{2}$ & $3 d: 872$ & $365 \times 150^{b}, 330^{k}$ & 44.01 & 77 \\
$\mathrm{Ar}^{*}-\mathrm{CH}_{4}$ & $4 p: 680,3 d: 872$ & $250^{b}, 380^{k}$ & 16.04 & 81,56 \\
$\mathrm{Ar}^{*}-\mathrm{C}_{2} \mathrm{H}_{6}$ & $4 s: 498$ & $400 \times 290^{b}, 380-400^{k}$ & 30.07 & 130 \\
$\mathrm{Ar}^{*}-\mathrm{C}_{3} \mathrm{H}_{8}$ & $4 s: 498$ & $500 \times 290^{b}, 430^{k}$ & 44.096 & 130 \\
$\mathrm{Ar}^{*}-\mathrm{iC}_{4} \mathrm{H}_{10}$ & $4 s: 498$ & $445 \times 555^{b}, 500-550^{k}$ & 58.123 & 100 \\
$\mathrm{Ar}^{*}-\mathrm{C}_{2} \mathrm{H}_{2}$ & $4 s: 498$ & $400 \times 150^{b}, 330^{k}$ & 26.04 & 150 \\
\hline $\mathrm{Xe}^{*}-\mathrm{Xe}$ & $4 f: 1850$ & $206^{m}, 260-280^{c}, 396^{k}$ & 131.293 & 37 \\
\hline
\end{tabular}

bond would have, or the dimensions computed from the bond lengths and bond angles of the molecule to be ionised. This type of diameter is also plausible for exchange processes in which overlap of the electron clouds is required. Kinetic diameters are the smallest separations molecules free to rotate can reach without force. They can be seen as the diameter of the smallest pores that let the molecules pass. Such definitions would e.g. be appropriate for dipole-dipole coupling. Other common definitions, such as the collision and van der Waals diameters are derived from macroscopic measurements and are not used in this report.

Excited atoms are larger than atoms in their ground state. The Deutsch-Märk electron-impact ionisation diameters for $\operatorname{Ar} 3 p^{5} 4 s, 4 p$ and $3 d$ are 3.6, 4.9 and 6.3 times the equivalent ground state, which coincides with the diameter of the maximum of the Dirac-Fock outer orbital. LennardJones calculations [86] (not for the potential minimum) put the $\operatorname{Ar} 3 p^{5} 4 s$ and $\operatorname{Ar} 3 p^{5} 4 p$ diameters at $1180 \mathrm{pm}$ and $1900 \mathrm{pm}, 2.2$ and 3.5 times larger than the equivalent ground state. For want of a better measure, we use the Deutsch-Märk and Hartree-Fock diameters of the excited states.

An incorrect choice of diameter biases collision-ionisation probabilities like $f_{B^{+}}$. However, the time-scale of the process, which is determined relative to the decay times of the excited states, would not be affected. Similarly, the net Penning transfer is not correlated with the diameters.

\subsection{Argon spectroscopy}

The principal argon excited states are (figure 3):

$3 \boldsymbol{p}^{\mathbf{5}} \mathbf{4}$ : The 4 lowest argon levels are located at $11.55 \mathrm{eV}, 11.62 \mathrm{eV}, 11.72 \mathrm{eV}$ and $11.83 \mathrm{eV}$ above the ground state. The lowest and third-lowest, $\operatorname{Ar}^{*}\left({ }^{3} \mathrm{P}_{0}\right)$ and $\operatorname{Ar}^{*}\left({ }^{3} \mathrm{P}_{2}\right)(J=0,2)$, are metastable with a lifetime of seconds. The $J=1$ levels, $\operatorname{Ar}^{*}\left({ }^{3} \mathrm{P}_{1}\right)$ and $\operatorname{Ar}^{*}\left({ }^{1} \mathrm{P}_{1}\right)$ mix. The lower level decays after $8.6 \pm 0.4 \mathrm{~ns}$ [56] under emission of a VUV photon. This is in agreement with calculations [33] but contradicts an earlier measurement giving $21 \pm 2 \mathrm{~ns}$ [68]. 


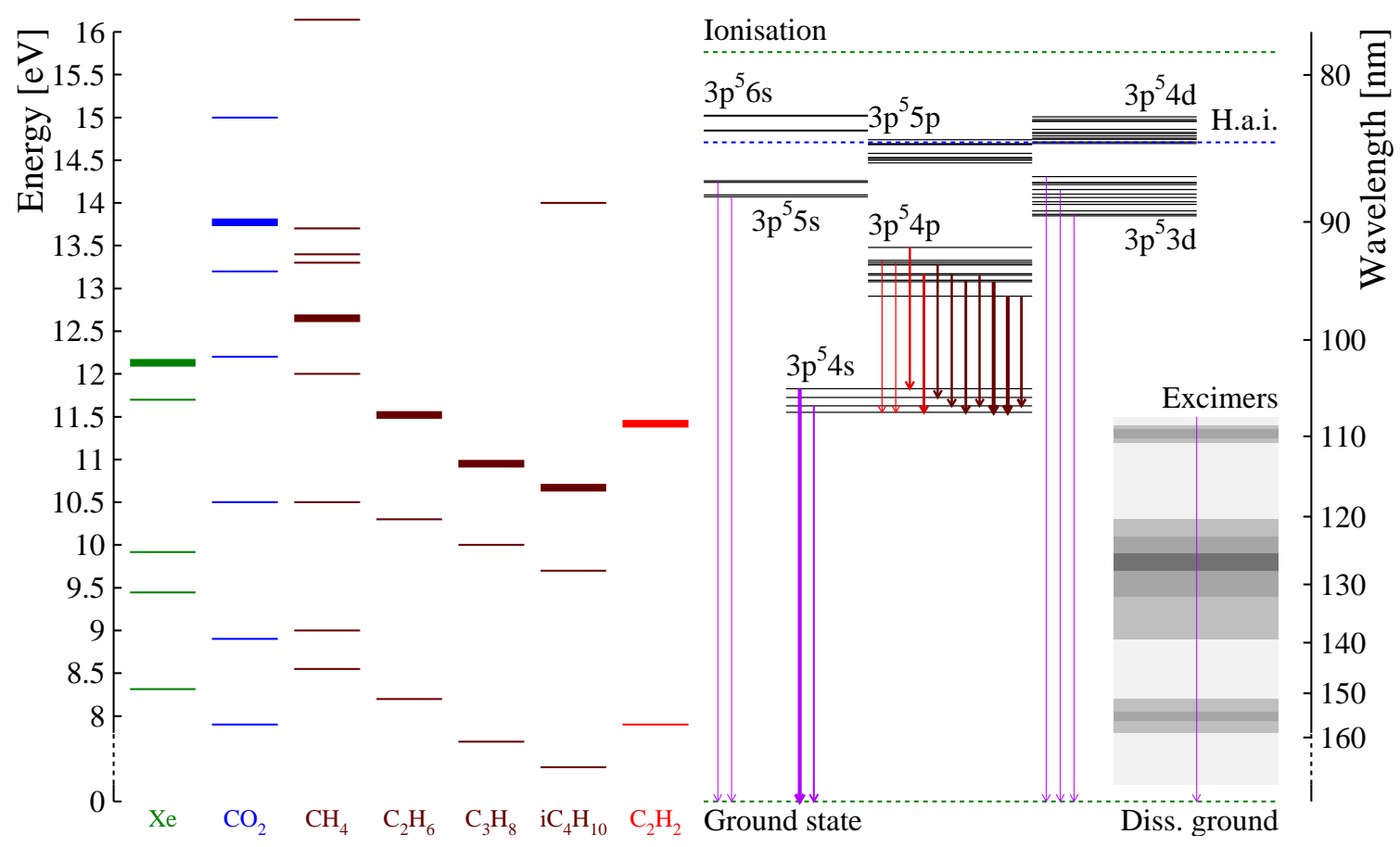

Figure 3. Left: excitation (thin) and ionisation (thick) levels of the admixtures studied in this paper. Not all levels are shown. Right: levels and transitions for the lowest excited states in neutral argon. Energy levels have been taken from [71]. Transitions in the VUV are shown in purple, visible lines are shown with their approximate colour while IR lines are brown. The width of the arrows is proportional to the line-intensity in discharges [70]. The grey-shading of the excimer spectrum is schematic because the intensity depends on the pressure and excitation source. The third continuum falls below the energy scale of the diagram.

Such discrepancies are common among the early publications, presumably as a result of "radiation trapping" whereby the energy emitted is re-absorbed [37, 38], and of collisional transfer with the nearby metastable states [5]. These effects depend on the pressure and increase the apparent lifetime. The higher $J=1$ level has a lifetime of $2.2 \pm 0.2 \mathrm{~ns}$ [56].

$\mathbf{3} \boldsymbol{p}^{\mathbf{5}} \mathbf{4} \boldsymbol{p}$ : These 10 levels, about $13 \mathrm{eV}$ above the ground state, predominantly decay into $3 p^{5} 4 s$ states by emitting red or infrared light $(697-912 \mathrm{~nm})$. The lifetime is in the range $21.7-$ $40.5 \mathrm{~ns}$ [91]. A non-resonant radiation trapping mechanism that affects the lifetimes of these levels has been reported [32]. Also observed in neon mixtures [27, 67], the impact is considerably smaller than for $3 p^{5} 4 s$.

$3 p^{\mathbf{5}} \mathbf{3 d}$ : In the mixtures we study here, these states are produced in amounts comparable to $3 p^{5} 4 p$. They have a threshold at $13.85 \mathrm{eV}$ and their lifetimes cluster around $50 \mathrm{~ns}$, with the exception of the $J=1$ levels which have an estimated lifetime of $\approx 3.5$ ns [33] because transition to the ground state is allowed.

higher levels: These levels, less frequently excited in avalanches, have lifetimes of $200-360 \mathrm{~ns}$ for $3 p^{5} 4 d, 100-210 \mathrm{~ns}$ for $3 p^{5} 5 p$ [51], $\approx 75 \mathrm{~ns}$ for $3 p^{5} 6 s$ [16] and $250-300 \mathrm{~ns}$ for $3 p^{5} 6 p$ [4]. 


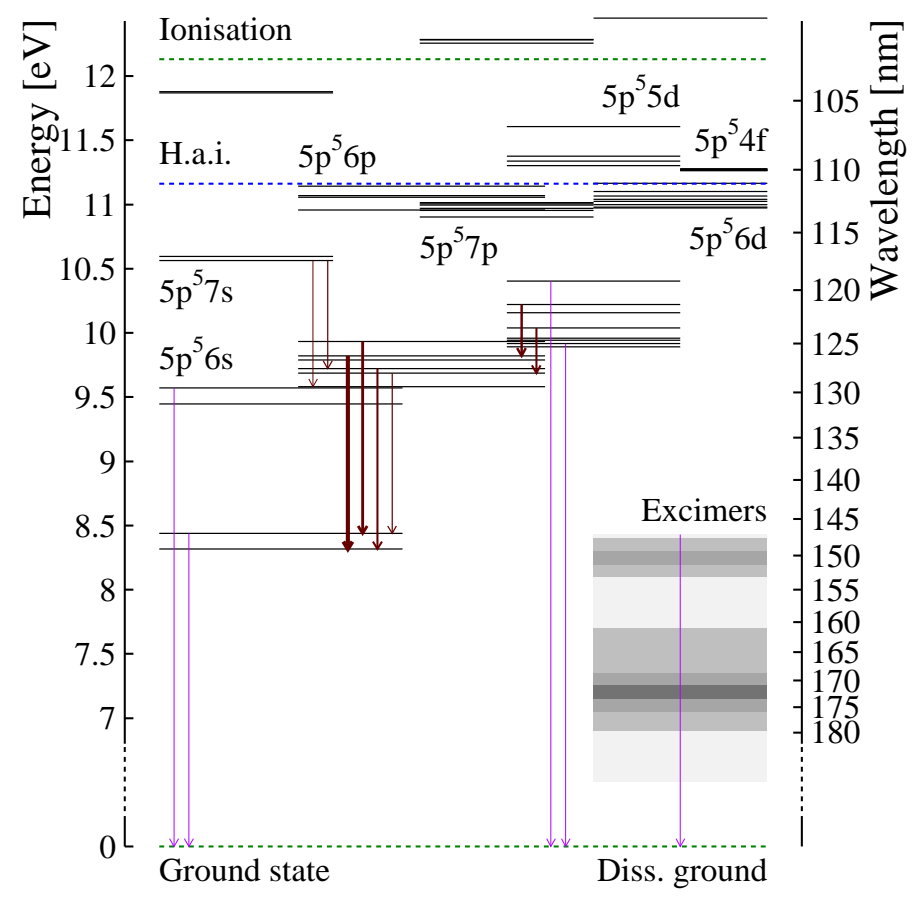

Figure 4. Simplified level diagram for xenon. Energy levels have been taken from [78]. Transitions in the VUV are shown in purple, visible lines are shown with their approximate colour while IR lines are brown. The width of the arrows is proportional to the line-intensity in discharges [70]. The grey-shading of the excimer spectrum is schematic because the intensity depends on the pressure [95].

\subsection{Xenon spectroscopy}

Xenon, like argon, has two metastable states amongst its four $5 p^{5} 6 s$ states. These have a lifetime of several seconds [89]. The two $J=1$ states mix, like in argon, but $\tau\left({ }^{3} \mathrm{P}_{1}\right)=3.79 \pm 0.12 \mathrm{~ns}$ and $\tau\left({ }^{1} \mathrm{P}_{1}\right)=3.17 \pm 0.19$ ns have been reported [8]. Various $5 p^{5} 6 p$ levels have been found to have lifetimes in the range $36-47$ ns [79]. The $5 p^{5} 4 f$ level, like $5 p^{5} 6 p$ near the associative ionisation threshold, has a lifetime of $55 \mathrm{~ns}$ [26]. The $5 p^{5} 7 p$ states have a lifetime of $100-120 \mathrm{~ns}$ [39].

\subsection{Argon excimers}

Excimer radiation has a rich structure (figure 3):

$110 \mathrm{~nm}(\mathbf{1 1 . 3} \mathrm{eV})$ : The "first continuum", a peak located just below the argon excited levels, is thought to correspond to transitions from high vibrational levels onto a geometrically wellseparated dissociative ground state [66]. At high pressure, the vibrational levels lose their energy rapidly.

$120-140 \mathrm{~nm}(\mathbf{8 . 9}-\mathbf{1 0 . 3} \mathrm{eV})$ : Peaking at $126 \mathrm{~nm}(9.8 \mathrm{eV})$, the "second continuum" is a dominant feature of excimer spectra. It is attributed to decays from lower vibrational levels.

$155 \mathrm{~nm}(\mathbf{8 . 0} \mathrm{eV})$ : The "left-turn point" is a narrow cusp resulting, like the first continuum, from the decay of high vibrational levels, but into a dissociative ground state where the argon 
atoms are near each other [92]. This feature, like the first continuum, disappears with increasing pressure.

$180-230 \mathrm{~nm}(5.4-6.9 \mathrm{eV}))$ : This "third continuum" has been observed over a broad range of pressures and with various excitation sources. Its mechanism has been the subject of much debate. One explanation is that this involves the dissociation of a bound state of a doublyionised argon atom and a neutral argon atom: $\left(\mathrm{Ar}^{++} \mathrm{Ar}\right) \rightarrow \mathrm{Ar}^{+}+\mathrm{Ar}^{+}$[54].

Excimer radiation is not expected to ionise the admixtures we study here: the photons with the highest energy come from the first continuum at $11.3 \mathrm{eV}$, above the ionisation potentials of $\mathrm{C}_{3} \mathrm{H}_{8}$ and $\mathrm{iC}_{4} \mathrm{H}_{10}$, but this source is pressure-suppressed. Radiation from the (dominant) second continuum at $9.8 \mathrm{eV}$ can ionise admixtures that have a particularly low ionisation potential (e.g. DME, dimethoxy-methane and cyclo-propane), but none of the admixtures we consider here. Xenon and the alkanes, but not argon and $\mathrm{CO}_{2}$, absorb such photons efficiently, with a mean free path at $1 \mathrm{~atm}$ of $\lambda \leq 15 \mu \mathrm{m}$ (section 2.6). In the unlikely case that these photons reach a cathode, photon feedback can occur (section 3.3). The remainder of the excimer spectrum is even lower in energy.

For similar reasons, the excimers are not likely to ionise admixtures through collisions. The most noticeable effect of excimers that remains is the removal of excited states.

\subsection{Associative ionisation}

Homonuclear associative ionisation in argon can occur for the highly excited states since the energy threshold for this process is $14.710 \pm 0.009 \mathrm{eV}$ [17, 43], well below the ionisation energy and approximately at the level of argon $3 p^{5} 5 p$ excitations. Contributions from the $3 p^{5} 3 d$ and $3 p^{5} 5 s$ states seem improbable because the mean kinetic energy of Ar molecules $\left(\frac{3}{2} k_{\mathrm{B}} T_{\mathrm{gas}}=0.04 \mathrm{eV}\right)$ is an order of magnitude smaller than the energy gap $(0.5-1 \mathrm{eV})$.

The process also exists in xenon [55] where it has a threshold of $11.162 \pm 0.005 \mathrm{eV}$ [43]. Xe has close to this threshold numerous excitation levels which can contribute to the process.

Heteronuclear associative ionisation has been observed for nearly all pairs of noble gases. $\mathrm{ArXe}^{+}$has a production threshold of $13.5 \pm 0.1 \mathrm{eV}[69]$.

\subsection{UV absorption}

Argon is virtually transparent to UV photons below $\approx 10.5 \mathrm{eV}$. It has a series of discrete UV absorption lines between $11 \mathrm{eV}$ and the ionisation potential. In this region, the cross section rapidly oscillates, reaching a maximum of $580 \mathrm{Mb}$ at the energy of the higher $3 p^{5} 4 s J=1$ level. The gas is transparent in-between peaks. The continuum photo-absorption cross section is $25-40 \mathrm{Mb}$ for photon energies between the ionisation potential and $30 \mathrm{eV}$ [22].

Xenon has a significantly higher photo-absorption cross section than argon and in addition absorbs photons in discrete lines down to lower energies $(\approx 8 \mathrm{eV})$.

Methane [11] is transparent below $\approx 8.5 \mathrm{eV}$ and the photo-absorption cross section rises via a number of absorption lines to $50 \mathrm{Mb}$ at the ionisation potential. Heavier alkanes follow the same pattern with progressively higher photo-absorption cross sections. The energy at which maximum absorption occurs increases, while the lowest ionisation potentials fall. Virtually no data for isobutane was found in the literature, but there is unconfirmed evidence [63] that isobutane is a better absorber still than n-butane for which data is available. 

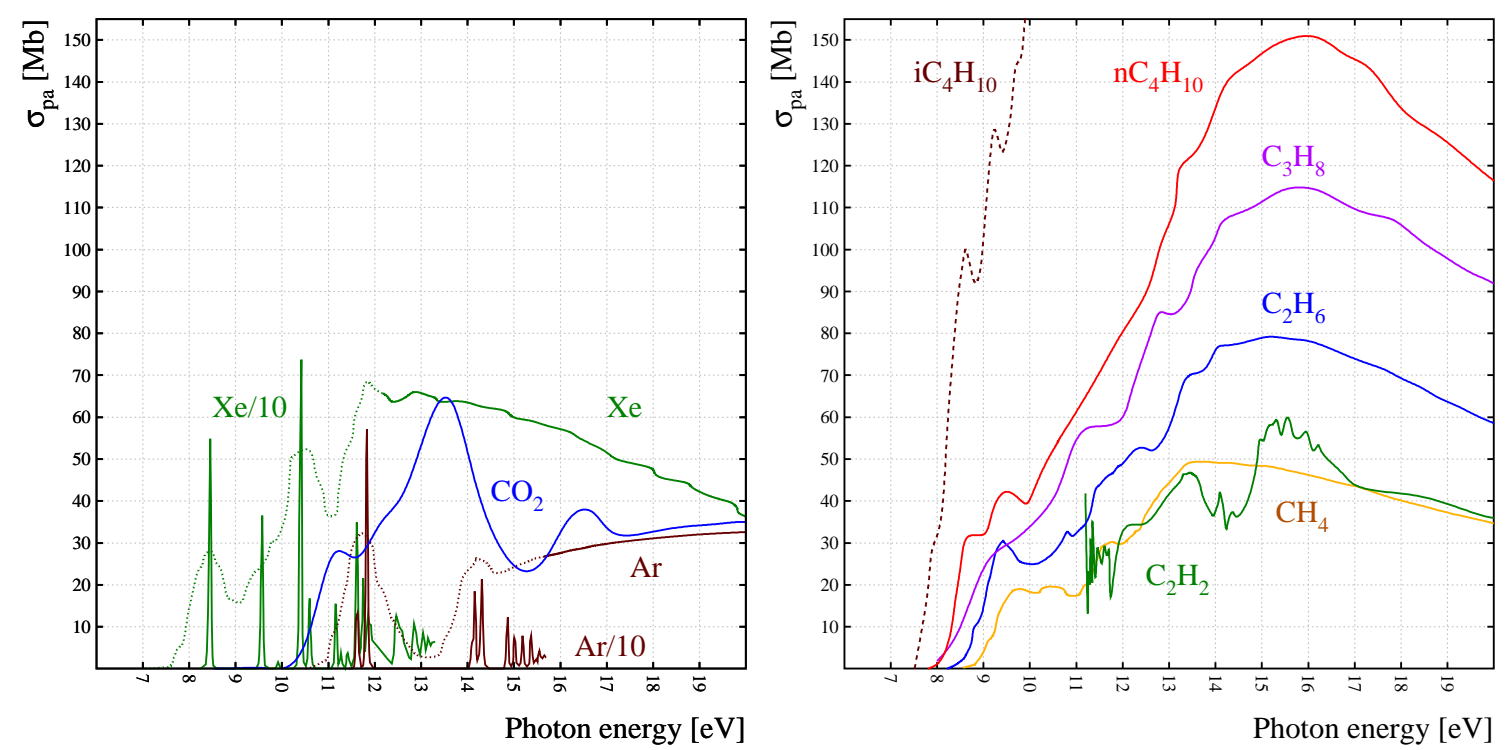

Figure 5. Photo-absorption cross section for argon and the admixtures considered in this paper. Compiled from the review of J. Berkowitz [11] and references therein, J.W. Au et al. [10], W.F. Chan et al. [22, 23], Y. Hatano et al. [35], K. Kameta et al. [48] and B.A. Lombos et al. [61-63]. In the discrete part of the argon and xenon spectra, dotted lines show the averaged cross sections and solid lines the scaled-down high-resolution data from W.F. Chan [21].

Although $\mathrm{CO}_{2}$ absorbs photons from $\approx 7.5 \mathrm{eV}$ onwards, the cross section becomes significant only at $\approx 10 \mathrm{eV}$. The gas has a rich structure of narrow absorption bands, not shown in detail in figure 5 , up to $\approx 19 \mathrm{eV}[53,82]$.

\section{Transfer probability measurement}

\subsection{Townsend coefficient adjustment}

Limiting ourselves to pressures that are sufficiently high for equilibrium transport equations to be applicable, we can measure the transfer probabilities $r_{i}$ by fitting gas gain curves with a Townsend coefficient corrected for excitation-induced ionisation:

$$
G=\exp \int_{\text {tube }}^{\text {anode }} \mathrm{d} r \alpha(E(r)) \frac{\sum v_{i}^{\text {ion }}(E(r))+\sum r_{i} v_{i}^{\text {exc }}(E(r))}{\sum v_{i}^{\text {ion }}(E(r))}
$$

The sum of the ionisation frequencies $v_{i}^{\text {ion }}$ is assumed proportional to the unadjusted Townsend coefficient $\alpha$. Only the excitation frequencies $v_{i}^{\mathrm{exc}}$ of states eligible for transfer are considered. Both frequencies can be computed by the Magboltz program [14]. The transfer rates $r_{i}$ are expected to depend on the gas pressure $p$ and the mixing proportions $c$ as discussed in section 1.3.

Various adjustments of the gain and the Townsend coefficient need to be considered:

\subsection{Gain calibration}

$$
G:=g G
$$


Some measurements $[24,94]$ are subject to considerable uncertainty (up to a factor of 10) in the absolute gain - the relative gain is generally known with more accuracy. Reasons advanced for this include uncertainty in the work function and calibration of the equipment.

The gain scaling factor $g$ is correlated with the transfer rates $r_{i}$ in that both $g$ and $r_{i}$ increase the measured gain. Fortunately, the electric field dependence of the excitation and the ionisation frequencies differs and the gain curves contain sufficient information to disentangle $g$ and $r_{i}$.

We always use a common $g$ for all anode voltages, and whenever possible also for all pressures and mixtures of a set of measurements. We first do a joint fit of $r_{i}$ and $g$ and then redo the fits fixing $g$ at the weighted average, leaving only $r_{i}$ free. The errors on $r_{i}$ are corrected using the correlation coefficient with $g$, or by determining the $g$-interval over which $\chi^{2}$ of the $r_{i}$-fit changes by 1 unit, and translating this $g$-interval into an $r_{i}$-interval.

\subsection{Photon feedback}

$$
G:=G /(1-\beta G)
$$

Avalanches produce photons which are, depending on the transparency of the gas, absorbed within the avalanche, in the gas outside the avalanche or at the cathode. In the first case, the photon contributes to the avalanche via the $f_{\text {rad }}$ term. In the two latter cases, the photon can cause a secondary avalanche, called feedback. If an avalanche of $n$ electrons produces on average $\beta n$ secondary avalanches, then the combined size will be $n+\beta n^{2}+\beta^{2} n^{3}+\cdots=n /(1-\beta n)$, see also [18]. The statistical fluctuations of feedback have been calculated by Werner Legler [57, 58].

Continuous current gain measurements do not distinguish secondary from primary avalanches. Photon feedback is unmistakable however in the gain curves: the gain enhancement is negligible for low anode voltages and thus low gain, but increases with the gain as the anode voltage rises, until breakdown occurs when $\beta n=1$.

Since $\beta$ is not strongly correlated with the main parameter we wish to determine, the transfer rates $r_{i}$, we do not constrain $\beta$ in fits. We will report on $\beta$ in a forthcoming publication.

\subsection{Other effects}

It has been conjectured that the ionisation cross sections used by Magboltz for a few pure gases (Xe in particular) would be too high. Although one can attempt to correct for this by scaling the Townsend coefficient, such an approach is unsatisfactory since it is unlikely that the ionisation cross section would be uniformly too high. Calculating a differential scaling requires more detailed experimental data than we have access to.

Temperature variations change the density $N$ and hence the gain. The effect increases with the gain and gases at high pressure are most affected. For the experimental configurations considered in this paper, and $G<10^{4}$ :

$$
\frac{\delta G}{G}<14 \frac{\delta N}{N}
$$

A temperature variation of $\delta T_{\text {gas }}=5 \mathrm{~K}$ would e.g. lead to a gain variation $\delta G / G<23 \%$, which is smaller than the gain scaling which we will generally need. 

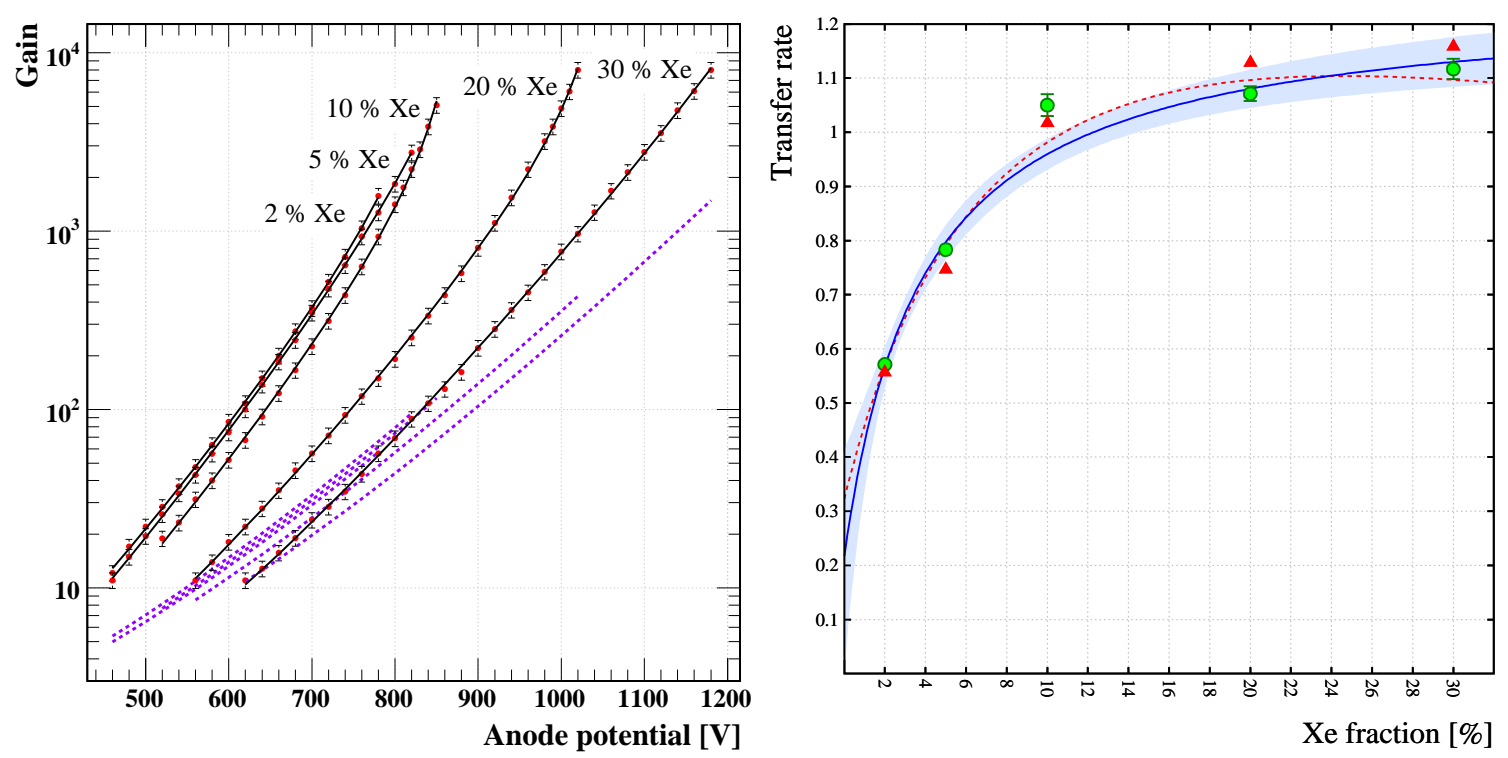

Figure 6. Left: Measured gain curves for Ar-Xe mixtures from P.C. Agrawal et al. [3] (red dots) and fits to obtain the transfer rates (black lines). For comparison, dashed purple lines show the calculated gain curves without transfer. Right: Transfer rates. The green circles show the rates obtained when leaving the gain scaling and photon feedback free. For the red triangles, the gain scaling has been constrained to a common value of 0.39 . The red curve shows the fit allowing for three-body reactions according to eq. (4.2). It is dashed to indicate that the fit parameters are unphysical. Such reactions are excluded for the blue fit. The blue error band shows the statistical uncertainty of the parametrisation.

\section{Experimental data}

\subsection{Argon-xenon mixtures}

P.C. Agrawal et al. [3] have measured the gain of argon with 2\%, 5\%, 10\%, $20 \%$ and $30 \%$ xenon at $p_{\text {gas }}=1 \mathrm{~atm}$ in a square $1 \times 1 \mathrm{~cm}^{2}$ tube with a $25 \mu \mathrm{m}$ diameter wire in the centre. In the vicinity of the wire, where multiplication occurs, the field is an excellent approximation of that found in a round tube with radius $r=0.54 \mathrm{~cm}$.

Photon feedback is small but visible when $G>10^{3}$, except for the $30 \%$ Xe mixture where the feedback parameter $\beta$ is not measurably different from zero (figure 6). Since $\beta$ is only weakly correlated with the transfer rates, we leave $\beta$ free in the fits. Gain rescaling is required with $g \approx 0.4$, varying by $4 \%$ between the mixtures (figure 7 ).

None of the xenon excited states comes close to the argon ionisation threshold. The argon $3 p^{5} 4 s$ levels are below the xenon ionisation threshold and are not considered when fitting the transfer rates, but we will discuss an indirect associative ionisation channel at the end of this section. All other argon excitations are eligible for direct transfer (figure 3). The most abundant of these states are, according to Magboltz 8.6, $3 p^{5} 4 p$ and $3 p^{5} 3 d$ which are produced in almost equal amounts, with almost identical electric field dependence (figure 7). We can not separate these two contributions by fitting the gain curves and we assume equal transfer probabilities for these and all higher levels. Furthermore, the quality of the data and the difference in lifetime are insufficient to separate the contributions in the model fit of $r(c)$. We therefore use an average value of $\tau_{A^{*}}$. 

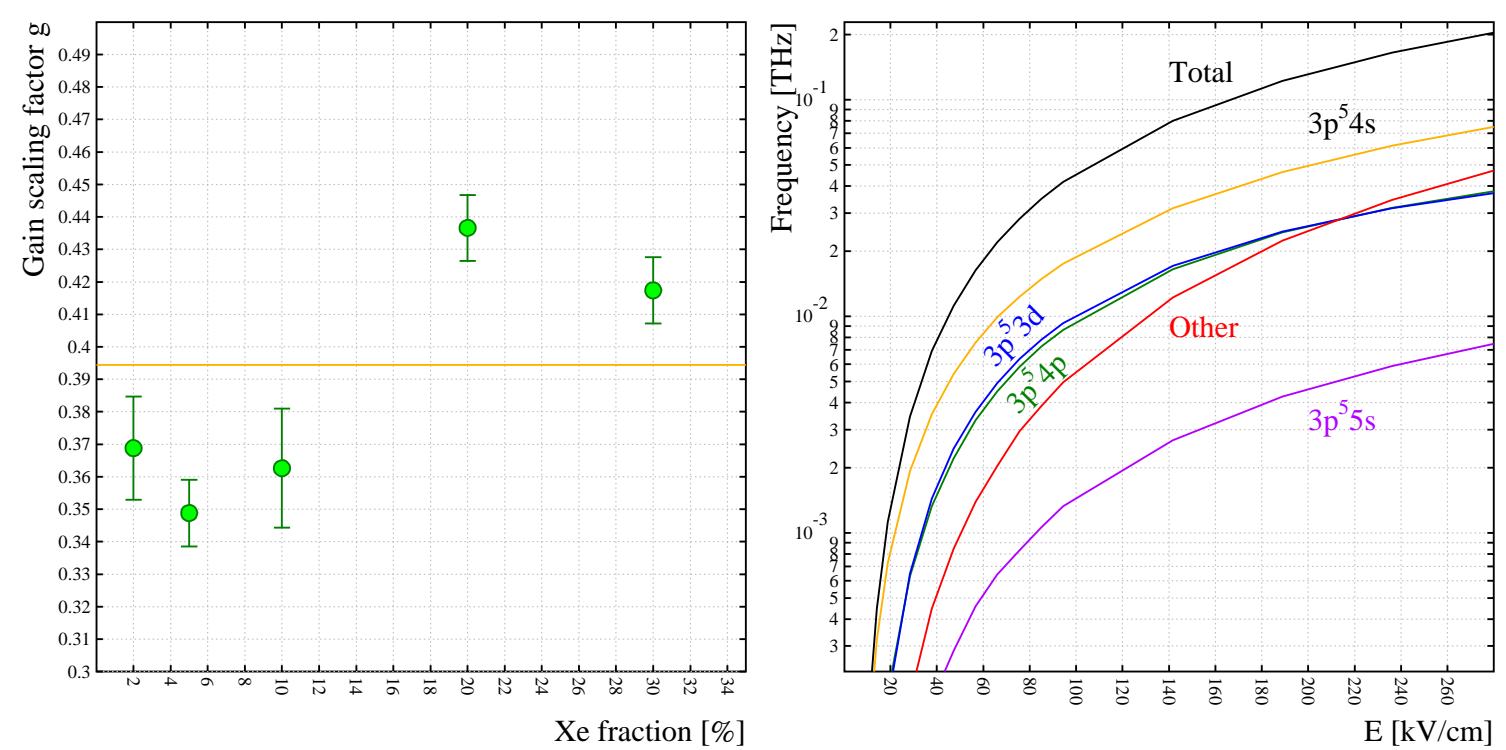

Figure 7. Left: Gain scaling factors that give the best transfer rate fits. The weighted average is shown by the orange line. Right: Production rates of argon excited states in argon with $20 \%$ xenon, the other mixtures are qualitatively similar.

Argon excimers are unlikely to ionise xenon (section 2.4), but they can contribute to the loss of excited argon states.

Radiation from the decay of $3 p^{5} 4 s$ states is not sufficiently energetic to ionise xenon and the $3 p^{5} 4 p$ states decay first to $3 p^{5} 4 s$. Remain the $3 p^{5} 3 d \rightarrow 3 p^{6}$ transitions at $13.86 \mathrm{eV}, 14.15 \mathrm{eV}$ and $14.31 \mathrm{eV}$, and the $3 p^{5} 5 s \rightarrow 3 p^{6}$ transitions at $14.09 \mathrm{eV}$ and $14.26 \mathrm{eV}$. At these energies, $\sigma_{\mathrm{pa}}^{\mathrm{Xe}} \approx 65 \mathrm{Mb}$. Photons can be re-absorbed in the discrete argon lines but this merely increases the apparent lifetime. As a result, the probability $f_{\text {rad }}$ that a photon ionises should be approximately independent of $c$, see eq. (1.15), so that eq. (1.13) can be expanded in $c$ as:

$$
r(c)=\frac{c\left[\frac{f_{B^{+}}}{\tau_{A^{*} B}}-\frac{f_{A^{+}}}{\tau_{A^{*} A}}\right]+\left[\frac{f_{A^{+}}}{\tau_{A^{*} A}}+\frac{f_{\mathrm{rad}}}{p \tau_{A^{*}}}\right]}{(1-c)^{2}\left[p \frac{f_{A_{2}}}{\tau_{A A A}}\right]+c\left[\frac{f_{B^{+}+}+f_{\bar{B}}}{\tau_{A^{*} B}}-\frac{f_{A^{+}+}+f_{\bar{A}}}{\tau_{A^{*} A}}\right]+\left[\frac{f_{A^{+}}+f_{\bar{A}}}{\tau_{A^{*} A}}+\frac{1}{p \tau_{A^{*}}}\right]}
$$

This prompts the use of a rational function to fit the transfer fraction:

$$
r(c)=\frac{a_{1} c+a_{3}}{a_{4}(1-c)^{2}+c+a_{2}}
$$

which, as shown in figure 6 , describes the data albeit with strongly correlated parameters, large error bars and values that are unphysical, e.g. $a_{2}$ would be negative. This is the result of having too few data points and too large uncertainties for the number of parameters. As a simplifying assumption, we exclude the loss of excited states through excimer formation:

$$
a_{1}=1.248 \pm 0.086, a_{2}=0.039 \pm 0.022, a_{3}=0.008 \pm 0.012, a_{4} \doteq 0
$$

Homonuclear associative ionisation can be presumed to be suppressed here because the data was taken at atmospheric pressure and because xenon in concentrations as small as $0.1 \%$ destroys 
the argon excited states (and argon excimers) by excitation energy transfer to xenon atoms [19]. The concentration-independent terms therefore give information on photo-ionisation. The photoionisation yield of xenon is thought to be $\eta^{\mathrm{Xe}} \approx 1$ from the ionisation threshold onwards [65]. According to Magboltz 8.6, the fraction of excited argon states that can undergo radiative decay ranges (depending on $c$ ) from $f_{\gamma}=0.27-0.29$ at $E=100 \mathrm{kV} / \mathrm{cm}$ to $f_{\gamma}=0.37-0.40$ at $E=200 \mathrm{kV} / \mathrm{cm}$. The resulting $f_{\text {rad }}$ is within error bars compatible with $a_{3} / a_{2}=0.22 \pm 0.19\left(a_{2}\right.$ and $a_{3}$ are strongly correlated).

From the value of $a_{2}$, the average lifetime of the excited states $\tau_{A^{*}}=30 \mathrm{~ns}(4 p), 50 \mathrm{~ns}(3 d)$ and the mean $\mathrm{Ar}^{*}$-Xe collision time $\tau_{A^{*} B}=130 \mathrm{ps}(4 p), 88 \mathrm{ps}(3 d)$, we find the efficiency of collisional energy transfer:

$$
f_{B^{+}}=\frac{1}{a_{2}} \frac{\tau_{A^{*} B}}{\tau_{A^{*}}}=0.11 \pm 0.06(4 p), 0.06 \pm 0.03(3 d)
$$

There are no constraints in the fitting procedure to limit the rate to $100 \%$ and the transfer rate actually exceeds $100 \%$ beyond $15 \%$ xenon. A transfer rate of $100 \%$ indicates that all excited argon atoms which do not undergo radiative decay, will eventually ionise a xenon atom through collisions. Losses through inelastic collisions are apparently not significant, which should come as no surprise since there are only noble gases in this mixture.

Transfer rates in excess of $100 \%$ can be the result of photo-ionisation occurring on average at a larger distance from the anode than the emission point, but the partial mean free path in xenon of the photons is only $\lambda=20 \mu \mathrm{m}$ for $c=0.3$. An error in the density can be ruled out since the equivalent of $p_{\text {gas }}=950$ mbar would be needed to find $a_{1}=1$. Conceivably also, the ionisation cross section could be too small. The Magboltz xenon cross sections are at the time of writing in the process of being updated and the possibility will be revisited once the update is complete.

Remains the transfer from $\operatorname{Ar} 3 p^{5} 4 s$ excited states, which would lead to a transfer probability in excess of 1 since we have normalised to the production rate of only those argon levels that are above the xenon ionisation threshold, i.e. Ar $3 p^{5} 4 p$ and higher. Given that $\operatorname{Ar} 3 p^{5} 4 s$ overlaps with Xe $5 p^{5} 4 f$ and $5 p^{5} 5 d$, which are located above the homonuclear associative ionisation threshold of xenon (sections 2.3 and 2.5), such transfers can produce ionisation electrons:

$$
\begin{aligned}
& \mathrm{Ar}^{*}+\mathrm{Xe} \rightarrow \mathrm{Ar}+\mathrm{Xe}^{*} \\
& \mathrm{Xe}^{*}+\mathrm{Xe} \rightarrow \mathrm{Xe}_{2}^{+}+e^{-}
\end{aligned}
$$

This process requires two collisions with xenon atoms. The first has to compete against the slow decay of $\operatorname{Ar} 3 p^{5} 4 s$ (section 4.3), the second against the decay of xenon levels above the homonuclear associative ionisation threshold. Since R.H. Lipson et al. found that homonuclear associative ionisation in xenon is more likely for $f$ than for $d$ states [60], the relevant lifetime is that of Xe $5 p^{5} 4 f$, i.e. 55 ns. One therefore does not expect this process to have a concentration dependence that is substantially steeper than collision and radiation transfer of $\operatorname{Ar} 3 p^{5} 4 p$ and higher. Since at $E=150-250 \mathrm{kV} / \mathrm{cm} \mathrm{Ar} 3 p^{5} 4 s$ excitations make up $\approx 40 \%$ of the total, the $10 \%$ excess observed in the $30 \%$ Xe mixture corresponds to $\mathrm{a} \approx 15 \%$ transfer probability for this process. $\mathrm{ArXe}^{+}$ associative ionisation could play a role too [72, 76], but this process has a threshold of $13.5 \mathrm{eV}$ (section 2.5), above the Xe ionisation level, and is therefore already accounted for. 

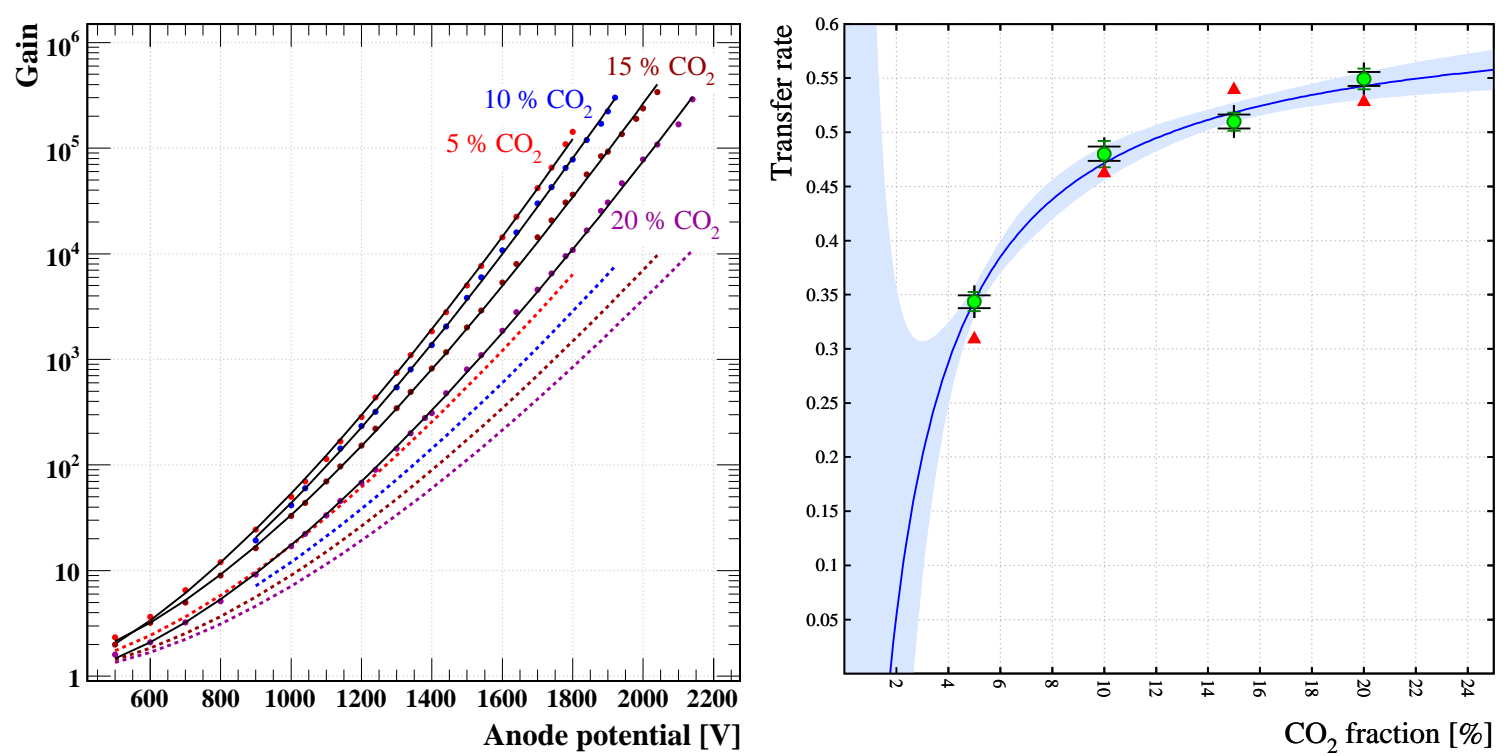

Figure 8. Left: Measured gain curves for $\mathrm{Ar}-\mathrm{CO}_{2}$ mixtures from T.Z. Kowalski et al. [52] (red dots) with fits of the transfer rates black lines). For comparison, dashed purple lines show the calculated gain curves without transfer. Right: Transfer rates fitted with $\left(a_{1} c+a_{3}\right) /\left(c+a_{2}\right)$ (blue curve) and the uncertainty on this parametrisation (blue error band). The $a_{3}$ parameter is outside the physical range (by $1.2 \sigma$ ). The larger error bars are obtained leaving gain scalings and transfer fractions free, while the broader and smaller error bars correspond to fits with fixed gain scalings. The triangles indicate the transfer rates found when using the weighted average of the gain scaling factor in all fits.

\subsection{Argon-carbon dioxide mixtures}

T.Z. Kowalski et al. [52] have measured the gain in argon with 5\%, 10\%, 15\% and $20 \% \mathrm{CO}_{2}$ using a rectangular $1.1 \times 1.6 \mathrm{~cm}^{2}$ tube with a $50 \mu \mathrm{m}$ diameter wire. The data has been taken at a precisely controlled pressure $p=1070 \mathrm{hPa}$ and temperature $T_{\text {gas }}=20^{\circ} \mathrm{C}$. In the vicinity of the wire, where multiplication occurs, the field is an excellent approximation of that found in a round tube with radius $r=0.67 \mathrm{~cm}$.

$\mathrm{CO}_{2}$ as a quencher is a good match for argon: $\mathrm{CO}_{2}$ has a cross section of $\approx 30 \mathrm{Mb}$ to stop radiation from the $3 p^{5} 4 s$ excitations, the $3 p^{5} 4 p$ states decay to $3 p^{5} 4 s$ under emission of (infra)red light, and the cross section is $\approx 60 \mathrm{Mb}$ at the $3 p^{5} 3 d$ level (figure 5). Decay photons will therefore be absorbed within the avalanche. Argon excimers could be a source of cathode feedback because photons from their radiative decay would not be absorbed in $\mathrm{Ar}-\mathrm{CO}_{2}$ mixtures: $\mathrm{CO}_{2}$ is transparent to $\mathrm{UV}$ photons below $10 \mathrm{eV}$. But there is no evidence for photon feedback in the gain curves, despite gains $G>10^{5}$ (figure 8), and we have not included feedback in the fits. The gain scaling factor is small, on average $g=1.06$, but it varies by more than $1 \sigma$ between mixtures. We leave $g$ free in the fits.

$\mathrm{CO}_{2}$, with its ionisation potential of $13.77 \mathrm{eV}$, opens a window on the higher argon excitations since only the states from $3 p^{5} 3 d$ upwards (lowest level at $13.85 \mathrm{eV}$ ) are eligible for transfer to $\mathrm{CO}_{2}$, through collisions and through absorption of decay photons. Fast radiative decay to the ground state of a few $3 p^{5} 3 d$ levels is allowed, the other levels have a lifetime in the range $40-90 \mathrm{~ns}$ (section 2.2). The excitation rates are virtually identical to those in Ar-Xe (figure 7). 
There is no indication that argon excited states are lost to excimer formation because the transfer rate shows no tendency to diminish at large $\mathrm{CO}_{2}$ concentrations, as discussed for $\mathrm{Ar}-\mathrm{Xe}$ mixtures (section 4.1). Excluding excimer terms, eq. (1.13) reduces to

$$
r(c)=\frac{c\left[\frac{f_{B^{+}}}{\tau_{A^{*} B}}-\frac{f_{A^{+}}}{\tau_{A^{*} A}}\right]+\left[\frac{f_{A^{+}}}{\tau_{A^{*} A}}+\frac{f_{\mathrm{rad}}}{p \tau_{A^{*}}}\right]}{c\left[\frac{f_{B^{+}}+f_{\bar{B}}}{\tau_{A^{*} B}}-\frac{f_{A^{+}}+f_{\bar{A}}}{\tau_{A^{*} A}}\right]+\left[\frac{f_{A^{+}}+f_{\bar{A}}}{\tau_{A^{*} A}}+\frac{1}{p \tau_{A^{*}}}\right]}=\frac{a_{1} c+a_{3}}{c+a_{2}}
$$

The ratio $a_{3} / a_{2}=-1.3 \pm 6.4$ which in principle contains information regarding photo-ionisation and ionisation through $\mathrm{Ar}^{*}$-Ar collisions, can for lack of transfer rates at low $\mathrm{CO}_{2}$ concentrations not be exploited (figure 8). Photo-ionisation should be present because radiative decay of many of the higher excited states of argon is allowed and because the photo-ionisation yield $\eta^{\mathrm{CO}_{2}}$ rises from $\approx 40 \%$ at the lowest $3 p^{5} 3 d$ levels to $\approx 80 \%$ at the highest levels [82, 87]. Homonuclear associative ionisation, which may matter in high-pressure $\mathrm{Ar}-\mathrm{CH}_{4}$ mixtures (section 4.4), should have less impact in this atmospheric pressure data.

The asymptotic transfer rate $a_{1}=0.620 \pm 0.057$ is significantly smaller than $100 \%$, i.e. excited states are lost, e.g. through inelastic collisions. $\mathrm{CO}_{2}$, as opposed to xenon, indeed has vibrational, rotational and polyad inelastic cross section terms. Further comparing with xenon (figure 6), one notes that the transfer probability levels off at a higher concentration of $\mathrm{CO}_{2}$. This is mainly the result of the longer lifetime of the excited states involved and of the smaller collision time. Using the average lifetime of $\tau_{A^{*}}=50 \mathrm{~ns}$ (section 2.2) and the mean collision time $\tau_{A^{*} B}=77 \mathrm{ps}$ of the $3 p^{5} 3 d$ states (table 1), and constraining $a_{3} \geq 0$ to find the lower limit $a_{2}>0.042$, we see that in addition the collisional ionisation and loss probabilities are lower:

$$
f_{B^{+}}+f_{\bar{B}}=\frac{1}{a_{2}} \frac{\tau_{A^{*} B}}{\tau_{A^{*}}}<0.04(84 \% \mathrm{CL})
$$

\subsection{Argon-ethyne mixtures}

Ar- $\mathrm{C}_{2} \mathrm{H}_{2}$ has one of the largest known Penning effects with a gain enhancement exceeding a factor of 100 for the optimal mixing proportion of $\approx 0.1 \% \mathrm{C}_{2} \mathrm{H}_{2}$ [36]. For this reason, these mixtures have been studied extensively, even though they do not have sufficient spark resistance for use in gas-based detectors. Historically, the strong Penning effect has been attributed to the proximity of the ionisation potential of $\mathrm{C}_{2} \mathrm{H}_{2}$ at $11.42 \mathrm{eV}$ and the energy of the argon $3 p^{5} 4 s$ states at $11.55 \mathrm{eV}$. These mixtures also owe part of their large Penning effects to the abundant production of excited states at low electric field strengths which results from the high electron energies typical for pure and nearly pure noble gases (figure 1). The Penning effect is largest in parallel plate chambers, where the $E$ fields are uniformly low, and is much reduced at the higher quencher concentrations used in gas-based detectors (figure 9).

Gain curves for argon with $0.5 \%, 2 \%, 5 \%$ and $10 \% \mathrm{C}_{2} \mathrm{H}_{2}$ at atmospheric pressure have been measured by P.C. Agrawal et al. [3] using a cylindrical chamber described in section 4.1. In addition, they have studied the $1 \%, 2 \%$ and $5 \%$ mixtures in a parallel plate detector made of a pair of $7 \mathrm{~cm}$ diameter stainless steel grids separated by a $1.07 \mathrm{~mm}$ gas gap [77]. J.P. Sephton et al. [80] have published a gain curve for $0.5 \% \mathrm{C}_{2} \mathrm{H}_{2}$ at $1.1 \mathrm{~atm}$ using a cylindrical chamber $\left(r_{\text {tube }}=11.7 \mathrm{~mm}, r_{\text {anode }}=12.7 \mu \mathrm{m}\right)$. 

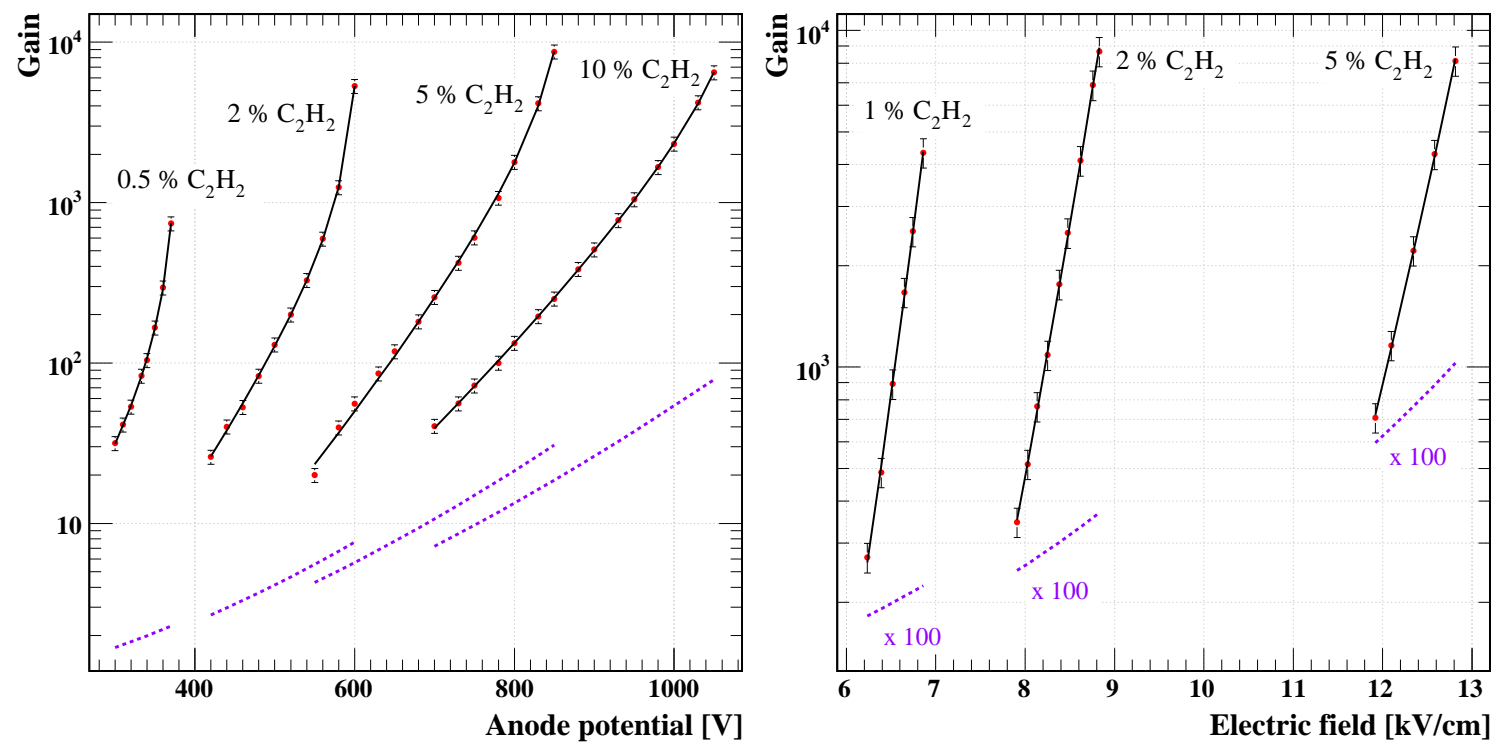

Figure 9. Measured gain curves for $\mathrm{Ar}-\mathrm{C}_{2} \mathrm{H}_{2}$ mixtures from P.C. Agrawal et al. [3] (red dots) and fits of the transfer rates. For comparison, dashed purple lines show the calculated gain curves without transfer. Black lines are fits assuming equal transfer rates for all excited states and leaving the gain scaling free. Cylindrical chamber data on the left, parallel plate chamber data on the right.

All gain curves for cylindrical chambers are affected by feedback. In contrast, the parallel plate chamber gain curves, even for $1 \% \mathrm{C}_{2} \mathrm{H}_{2}$ and $G>10^{4}$ are exponential (figure 9). This is discussed in [3]. P.C. Agrawal et al. caution that their gain may be overestimated by $20 \%$ due to uncertainty in the work function, but the parallel plate curves are best fitted with $g=0.21$ while we find $g=0.39$ for their cylindrical chamber data and $g=0.23$ for the J.P. Sephton et al. curve.

In $\mathrm{Ar}-\mathrm{C}_{2} \mathrm{H}_{2}$ mixtures, all excited states of argon are eligible for transfer. The parallel plate chamber data contains hints that the higher levels have a higher transfer rate, but since this evidence is at the limit of statistical significance, we assume all transfer rates are equal.

A striking feature of $\mathrm{Ar}-\mathrm{C}_{2} \mathrm{H}_{2}$ is that the transfer probabilities (figure 10) are approximately constant. Two factors may explain this: a high photo-ionisation probability and a long lifetime of the excited states $\tau_{A^{*}}$. The photo-ionisation probability in $\mathrm{C}_{2} \mathrm{H}_{2}$ is not higher than in the other gases we have studied, and the concentration is lower. But the excited states do have an unusually long lifetime. The most abundantly produced excitations are the two non-metastable $3 p^{5} 4 s$ levels and their average lifetime $\approx 5.4 \mathrm{~ns}$ is not compatible with the observed transfer rates. As pointed out in section $2.2, \tau_{A^{*}}$ is substantially larger than the natural lifetime because radiation from the nonmetastable states is trapped in the long-lived metastable states, both of which are located slightly below the non-metastable states. Most higher states have longer lifetimes.

The ionisation branching fraction of $\mathrm{C}_{2} \mathrm{H}_{2}$ in collisions with metastable excited argon, according to two different techniques, has been found to be $\Gamma_{Q^{+}}=k_{Q^{+}} / k_{Q}=0.61 \pm 0.14,0.74 \pm 0.07$ [47]. These values are to be compared with the asymptotic value $a_{1}$ of the transfer probability, equal to $f_{B^{+}} /\left(f_{B^{+}}+f_{\bar{B}}\right)$ if excimers and the radiative term are neglected, which is justified as seen above. In the cylindrical chamber $a_{1}=0.72 \pm 0.02$, while in the parallel plate chamber $a_{1}=0.67 \pm 0.02$ with a systematic uncertainty of \pm 0.1 . 

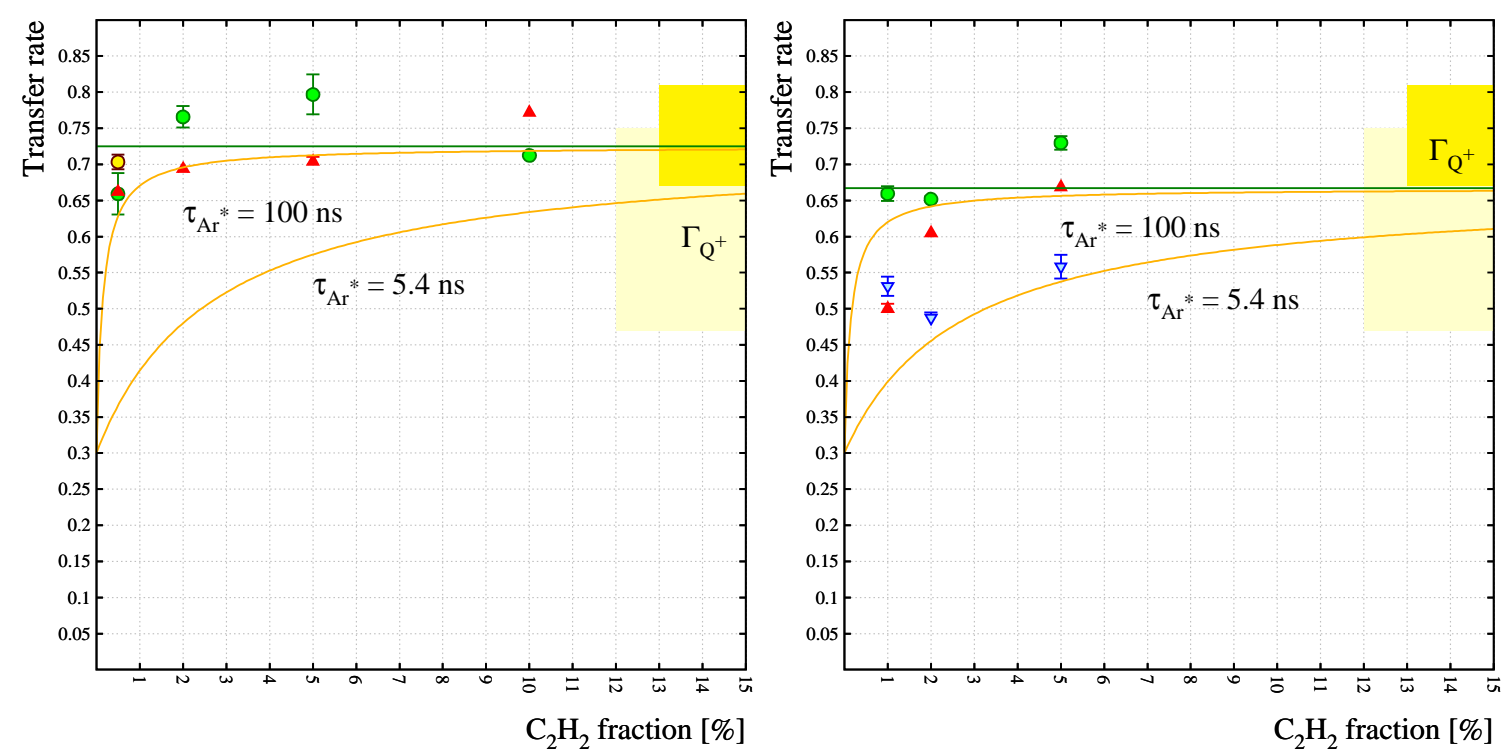

Figure 10. Transfer rates for $\mathrm{Ar}-\mathrm{C}_{2} \mathrm{H}_{2}$ in cylindrical chambers (left) and a parallel plate chamber (right). Orange lines: hypothetical excited states with a lifetime as indicated, $f_{\text {rad }}=0.3$ and $\tau_{A^{*} B}=150 \mathrm{ps}$, the collision time for $\mathrm{Ar}-\mathrm{C}_{2} \mathrm{H}_{2}$. The non-metastable $3 p^{5} 4 s$ argon excited states have an average lifetime of 5.4 ns. Green points: equal transfer rates for all excited states and unconstrained gain scaling, data from P.C. Agrawal et al. [3, 77]. Brown point: the same using the gain curve from J.P. Sephton et al. [80]. Green horizontal lines: average transfer rates. Red triangles: gain scaling fixed at the weighted average of all points. Blue down-pointing triangles: $3 p^{5} 4 p$ and higher states are fully transferred and gain scaling is fixed at the weighted average of the two highest concentrations. Error bands on the right: two measurements of the ionisation branching fraction, as discussed in the text.

\subsection{Argon-methane mixtures}

Z. Ye et al. [94] have measured gain curves in $\mathrm{Ar} 90 \% \mathrm{CH}_{4} 10 \%$ for $p_{\text {gas }}=1-21 \mathrm{~atm}$ and Ar $98 \% \mathrm{CH}_{4} 2 \%$ for $p_{\text {gas }}=1-28$ atm using a cylindrical counter $\left(r_{\text {tube }}=1.5 \mathrm{~cm}, r_{\text {anode }}=\right.$ $12.5 \mu \mathrm{m})$. Earlier, the same group [64], using a similar setup $\left(r_{\text {tube }}=1.25 \mathrm{~cm}\right)$, had performed measurements in $\mathrm{Ar} 95 \% \mathrm{CH}_{4} 5 \%$ and $\mathrm{Ar} 98 \% \mathrm{CH}_{4} 2 \%$ for $p_{\text {gas }}=350-2750 \mathrm{kPa}$. Further data at atmospheric pressure that we have used has been published by M.W. Charles [24] for $10 \% \mathrm{CH}_{4}$ using $10-100 \mu \mathrm{m}$ diameter anode wires inside a stainless steel tube $\left(r_{\text {tube }}=2.845 \mathrm{~cm}\right)$; by P.C. Agrawal et al. [1] for $2,5,10 \% \mathrm{CH}_{4}$ using the same equipment described in section 4.1; by S. Kishimoto et al. [50] for 2,10\% $\mathrm{CH}_{4}$ using a cylindrical chamber $\left(r_{\text {tube }}=2.5 \mathrm{~cm}, r_{\text {anode }}=25 \mu \mathrm{m}\right)$; by F. Tokanai et al. [85] for $10 \% \mathrm{CH}_{4}$ using a square $1 \times 1 \mathrm{~cm}^{2}$ counter and 5,10,20 $\mu \mathrm{m}$ diameter anode wires; and by A.H. Walenta et al. [88] for $10 \% \mathrm{CH}_{4}$ using a cylindrical counter $\left(r_{\text {tube }}=3 \mathrm{~mm}, r_{\text {anode }}=15 \mu \mathrm{m}\right)$.

The authors of [94] have gone to great lengths to normalise the gain correctly, investigating potential biases of several measurement techniques. The importance of this study becomes apparent when comparing the gain curve for $\mathrm{Ar} 98 \% \mathrm{CH}_{4} 2 \%$ with earlier data [64] from the same group, for the same gas and the same pressure, which is a factor $\approx 3$ lower. We use the newer data with a common scaling factor $g=1.25$. For the other data, common scaling factors $g=0.26-0.724$ have been used. There is no evidence of photon feedback, except in the $1 \mathrm{~atm}$ data of $\mathrm{Z}$. Ye et al. 

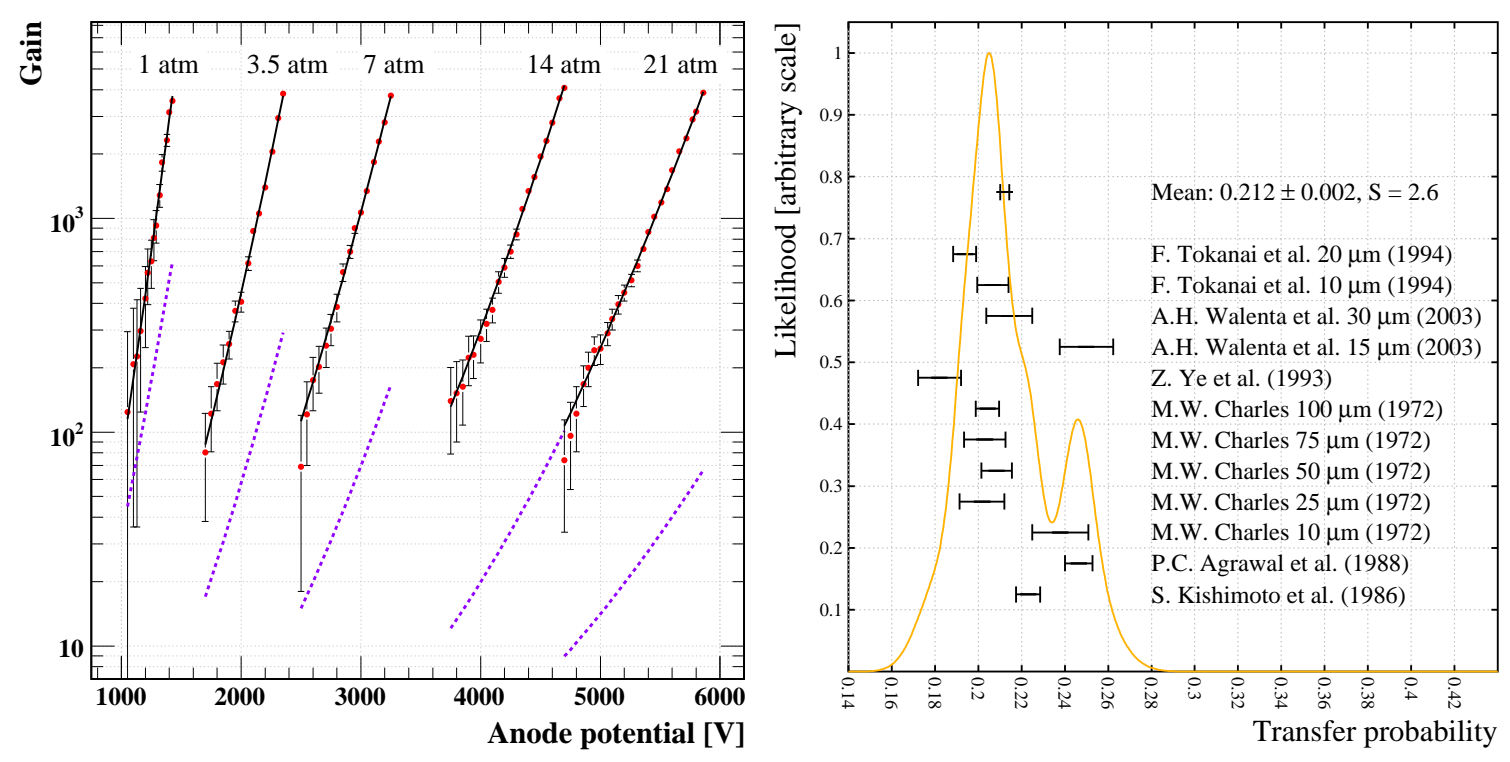

Figure 11. Left: Gas gain in $\mathrm{Ar} 90 \% \mathrm{CH}_{4} 10 \%$ measured by Z. Ye et al. [94], fitted with eq. (3.1) to obtain the Penning transfer probabilities. Red circles show the data, dashed purple lines show the gas gain computed from uncorrected Townsend coefficients and solid black lines show the fit with adjusted Townsend coefficients. Right: Compilation of transfer probability measurements in $\mathrm{Ar} 90 \% \mathrm{CH}_{4} 10 \%$ at atmospheric pressure. The spread of the transfer fraction measurements in $10 \% \mathrm{CH}_{4}$ at atmospheric pressure is $S=2.6$ with $S^{2}=\chi^{2} /($ ndf -1$)$. No excessive spread is seen in the $2 \% \mathrm{CH}_{4}$ atmospheric pressure data. In model fits, the weighted average and the error bar shown are used instead of the individual measurements.

The lowest argon excitation energetically eligible for transfer to $\mathrm{CH}_{4}$ (ionisation potential: $12.65 \mathrm{eV}$ ) is $3 p^{5} 4 p$. As for the xenon mixtures, $3 p^{5} 4 p$ and $3 p^{5} 3 d$ are produced in almost equal amounts and with a similar electric field dependence. Since we can not separate them in the fits, we assume these states have the same transfer probability. Except at high fields, the higher excitations are produced in smaller quantities. The data we have access to is not sufficient to identify their impact. Penning fits are shown in figure 11, the effect increases clearly with pressure.

We first look at the pressure dependence of the individual mixtures. As with the other mixtures considered here, argon excimers can not ionise $\mathrm{CH}_{4}$, but photons from radiative decays can. Expanded in $p$, eq. (1.13) reduces to:

$$
r(p)=\frac{p\left[c \frac{f_{B^{+}}}{\tau_{A^{*} B}}+(1-c) \frac{f_{A^{+}}}{\tau_{A^{*} A}}\right]+\left[\frac{f_{\text {rad }}}{\tau_{A^{*}}}\right]}{p^{2}\left[(1-c)^{2} \frac{f_{A_{2}}}{\tau_{A A A}}\right]+p\left[c \frac{f_{B^{+}}+f_{\bar{B}}}{\tau_{A^{*} B}}+(1-c) \frac{f_{A^{+}}+f_{\bar{A}}}{\tau_{A^{*} A}}\right]+\left[\frac{1}{\tau_{A^{*}}}\right]}
$$

The data is insufficient to determine all parameters for each of the mixtures separately. In particular, fits that include $f_{A_{2}}$ as free parameter are instable and the pressure dependence of the Ar $90 \% \mathrm{CH}_{4} 10 \%$ data can be described without this term. We therefore make the simplifying assumption that $f_{A_{2}} \doteq 0$, i.e. that excited states are not lost through excimer formation:

$$
r(p)=\frac{a_{1} p+a_{3}}{p+a_{2}}
$$



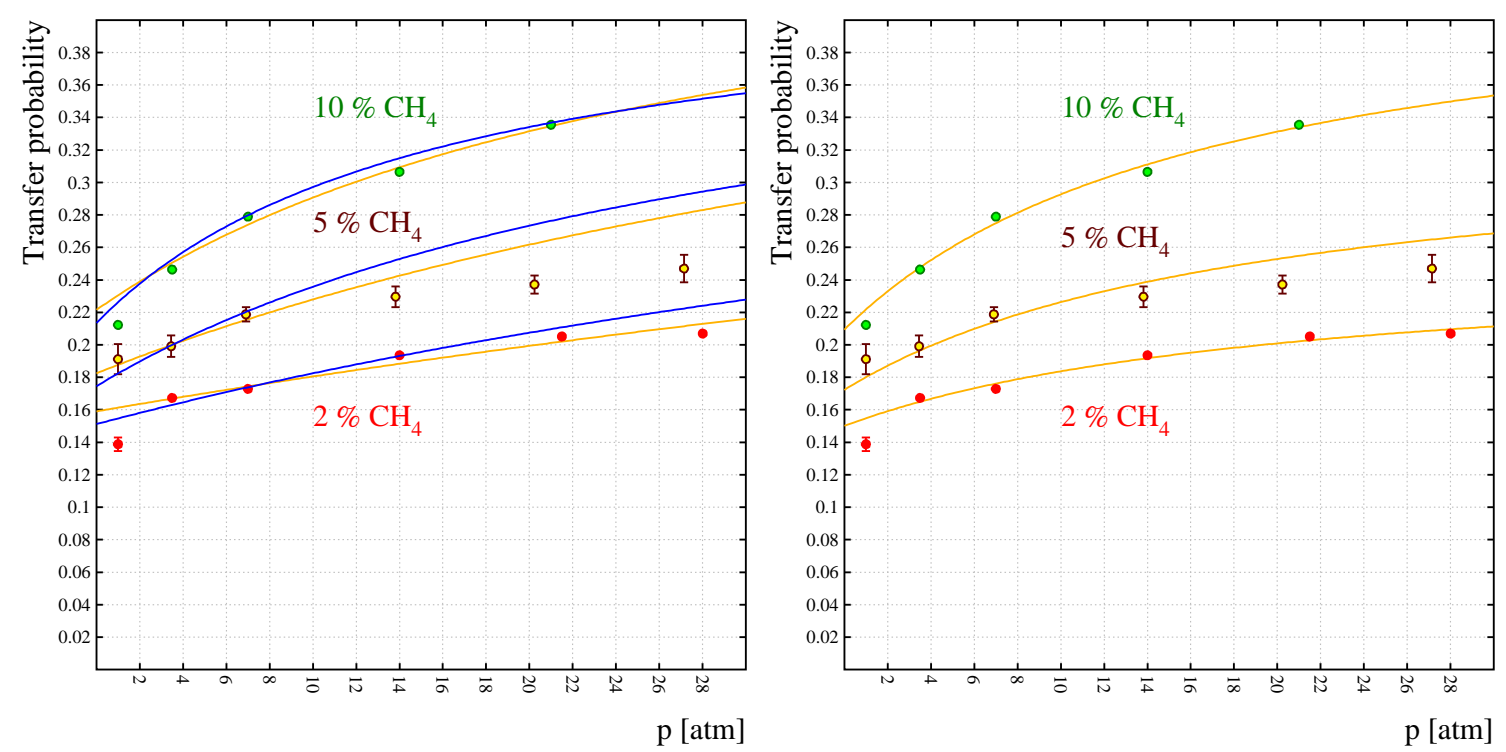

Figure 12. Transfer rates for $\mathrm{Ar} 90 \% \mathrm{CH}_{4} 10 \%$ (green dots), $\mathrm{Ar} 95 \% \mathrm{CH}_{4} 5 \%$ (brown dots) and Ar $98 \% \mathrm{CH}_{4} 2 \%$ (red dots). Left: In orange, a combined fit of all concentrations to eq. (4.12), under the constraint that Ar*-Ar collisions neither lead to losses nor to ionisation. The photo-ionisation efficiency $f_{\text {rad }}$ has an unconstrained linear dependence on the $\mathrm{CH}_{4}$ concentration. The blue lines are extrapolations of the eq. (4.10) fit of the $10 \%$ mixture to the $5 \%$ and $2 \%$ mixtures, assuming homonuclear associative ionisation does not occur and using a best-fit linear photo-ionisation efficiency. Right: The orange lines show a combined fit of all concentrations using eq. (4.12), which allows for homonuclear associative ionisation. The photo-ionisation efficiency $f_{\text {rad }}$ has in this fit an unconstrained linear dependence on the $\mathrm{CH}_{4}$ concentration.

The ratio $a_{3} / a_{2}=f_{\text {rad }}$ measures the ionisation of $\mathrm{CH}_{4}$ by photons from Ar* radiative decays such as $3 p^{5} 3 d \rightarrow 3 p^{6}$ and $3 p^{5} 5 s \rightarrow 3 p^{6}$. The energy of these photons ranges from $13.86 \mathrm{eV}$ almost to the ionisation potential. They can ionise since the photo-ionisation yield $\eta^{\mathrm{CH}_{4}}=\sigma_{\mathrm{pi}}^{\mathrm{CH}_{4}} / \sigma_{\mathrm{pa}}^{\mathrm{CH}_{4}}$ rises from $\eta=0$ at a photon energy of $13 \mathrm{eV}$ to $\eta=1$ at $16 \mathrm{eV}$ [48]. In this energy region, $\sigma_{\mathrm{pa}}^{\mathrm{CH}_{4}} \approx 50 \mathrm{Mb}$ [11], i.e. the partial mean free path at atmospheric pressure in $2-10 \% \mathrm{CH}_{4}$ is $375-75 \mu \mathrm{m}$ and proportionally less at higher pressure. These photons will therefore not reach the cathode. Absorption of photons in the discrete lines of argon should not lead to loss of excitation energy (section 1.3), hence $f_{\text {rad }}$ should in first approximation be independent of the concentration $c$, see eq. (1.15). Gain curves at higher $c$ have been measured at higher $E$ fields where the higher excited states are more populated. This implies that both $f_{\gamma}$ and the ionisation yield $\eta^{\mathrm{CH}_{4}}$ increase with $c$, and one therefore expects a slight increase of $f_{\text {rad }}$ with $c$, which is observed. Loss of the excitation energy by photo-absorption in argon, is ruled out by the data (figure 13).

The $a_{1}$ parameter reflects the efficiency with which excited argon atoms ionise other argon atoms and $\mathrm{CH}_{4}$ molecules through collisions:

$$
a_{1}=\frac{c f_{B^{+}} / \tau_{A^{*} B}+(1-c) f_{A^{+}} / \tau_{A^{*} A}}{c\left(f_{B^{+}}+f_{\bar{B}}\right) / \tau_{A^{*} B}+(1-c)\left(f_{A^{+}}+f_{\bar{A}}\right) / \tau_{A^{*} A}}
$$

The fit value for the $\operatorname{Ar} 90 \% \mathrm{CH}_{4} 10 \%$ mixture, $a_{1}=0.43 \pm 0.05$, indicates that loss collisions are nearly as frequent as ionising collisions. The asymptotic rates are $a_{1}=0.26 \pm 0.04$ for the $2 \%$ mixture and $a_{1}=0.27 \pm 0.04$ for the $5 \%$ mixture. 

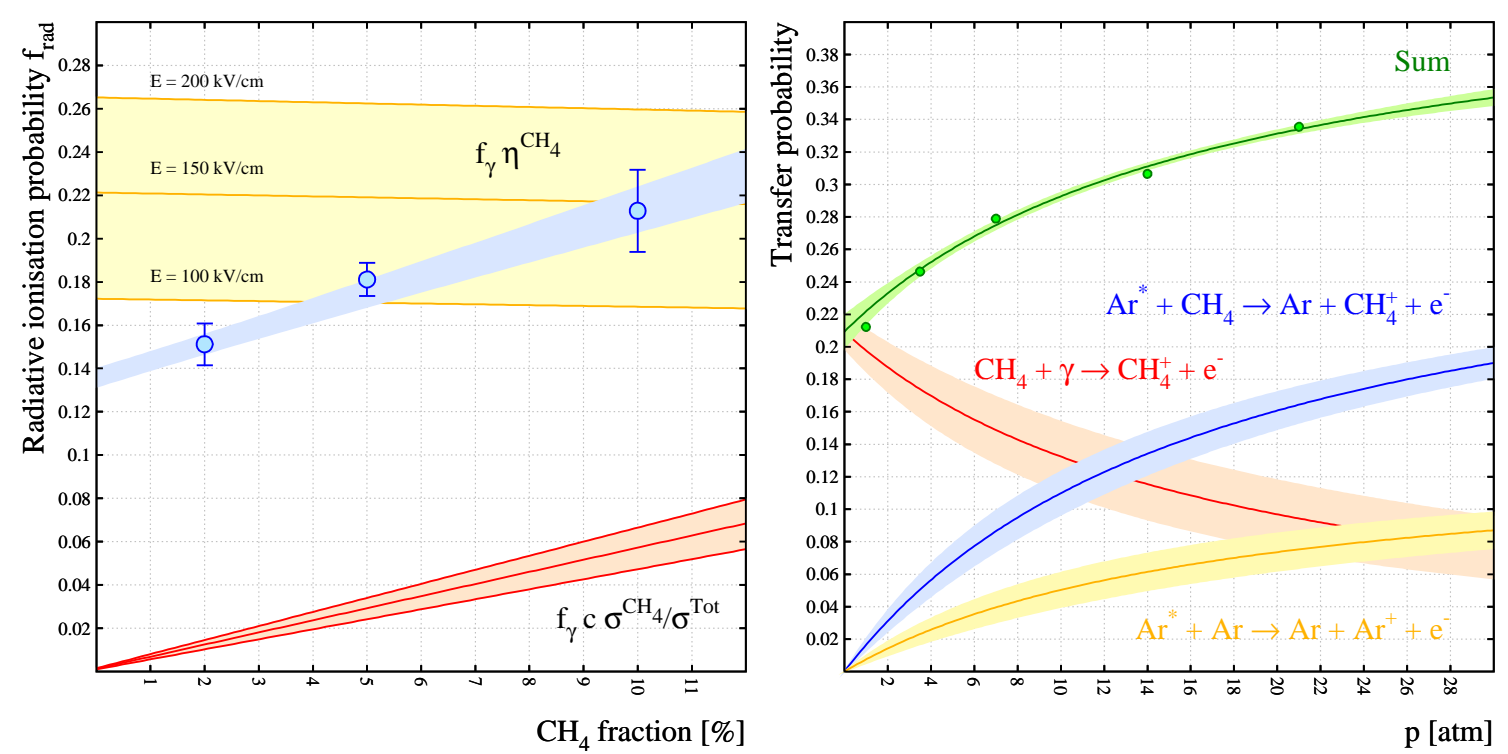

Figure 13. Left: $f_{\text {rad }}$ as measured for individual concentrations (blue error bars) and in a joint fit (blue error band), compared with eq. (1.14) for a weighted average of radiative states computed by Magboltz 8.6 at $E=100,150,200 \mathrm{kV} / \mathrm{cm}$, the UV absorption cross sections shown in section 2.6 and full ionisation yield (red error band), and with eq. (1.15) using the same weighted average of radiative states and the ionisation yield of K. Kameta et al. [48] (yellow band). Right: Contributions to the transfer rates in the 10\% mixture.

Ionisation through $\mathrm{Ar}^{*}$-Ar collisions can not be distinguished from ionisation through $\mathrm{Ar}^{*}-\mathrm{CH}_{4}$ collisions by studying the pressure dependence of the transfer probability at a single concentration, but this can be done by comparing several concentrations. If only $\mathrm{Ar}^{*}-\mathrm{CH}_{4}$ collisions, thought to be dominant, were to matter, then the high-pressure transfer rate should be the same for all mixtures. But, as seen above, $a_{1}$ is lower in the $2 \%$ and $5 \%$ mixtures than in the $10 \%$ mixture. Another hint of $\mathrm{Ar}^{*}$-Ar ionisation emerges when scaling the fit of the $\mathrm{Ar} 90 \% \mathrm{CH}_{4} 10 \%$ data to the other concentrations, setting $f_{A^{+}} \doteq f_{\bar{A}} \doteq 0$, or equivalently, when fitting with a 2-dimensional $(p, c)$ model based on eq. (1.13) without excimer and $\mathrm{Ar}^{*}$-Ar terms. Both procedures describe the data less well than a model in which $\mathrm{Ar}^{*}-\mathrm{Ar}$ collisions are allowed to ionise (figure 12). It should be noted that these observations depend to a large extent on a single set of high-pressure $5 \%$ data. Confirmation of these measurements would be welcome.

The Ar*-Ar contribution can be estimated with a model that allows both for ionisation through $\mathrm{Ar}^{*}-\mathrm{CH}_{4}$ collisions, which scales with $c$, and through $\mathrm{Ar}^{*}$-Ar collisions, which scales with $1-c$ :

$$
r(p, c)=\frac{b_{4} p c+b_{1} p(1-c)+b_{2} c+b_{5}}{b_{6} p c+p(1-c)+b_{3}}
$$

The parameters $b_{4}$ and $b_{6}$ are not sufficiently constrained for lack of data at high $\mathrm{CH}_{4}$ concentrations. Limiting them to the physical range $0 \leq b_{4} \leq b_{6}$, we find that $b_{4}=b_{6}$, i.e. $\mathrm{Ar}^{*}-\mathrm{CH}_{4}$ collisions are either elastic or ionising, but not inelastic. The fit is shown in figure 12.

The efficiency of Ar*-Ar ionisation $f_{A^{+}} /\left(f_{A^{+}}+f_{\bar{A}}\right)=b_{1}=0.20 \pm 0.01$ is stable under a variety of conditions, such as variations in the datasets considered and in the gain scaling. As illustrated in figure 13 for the $10 \%$ mixture, homonuclear associative ionisation of argon contributes at atmo- 

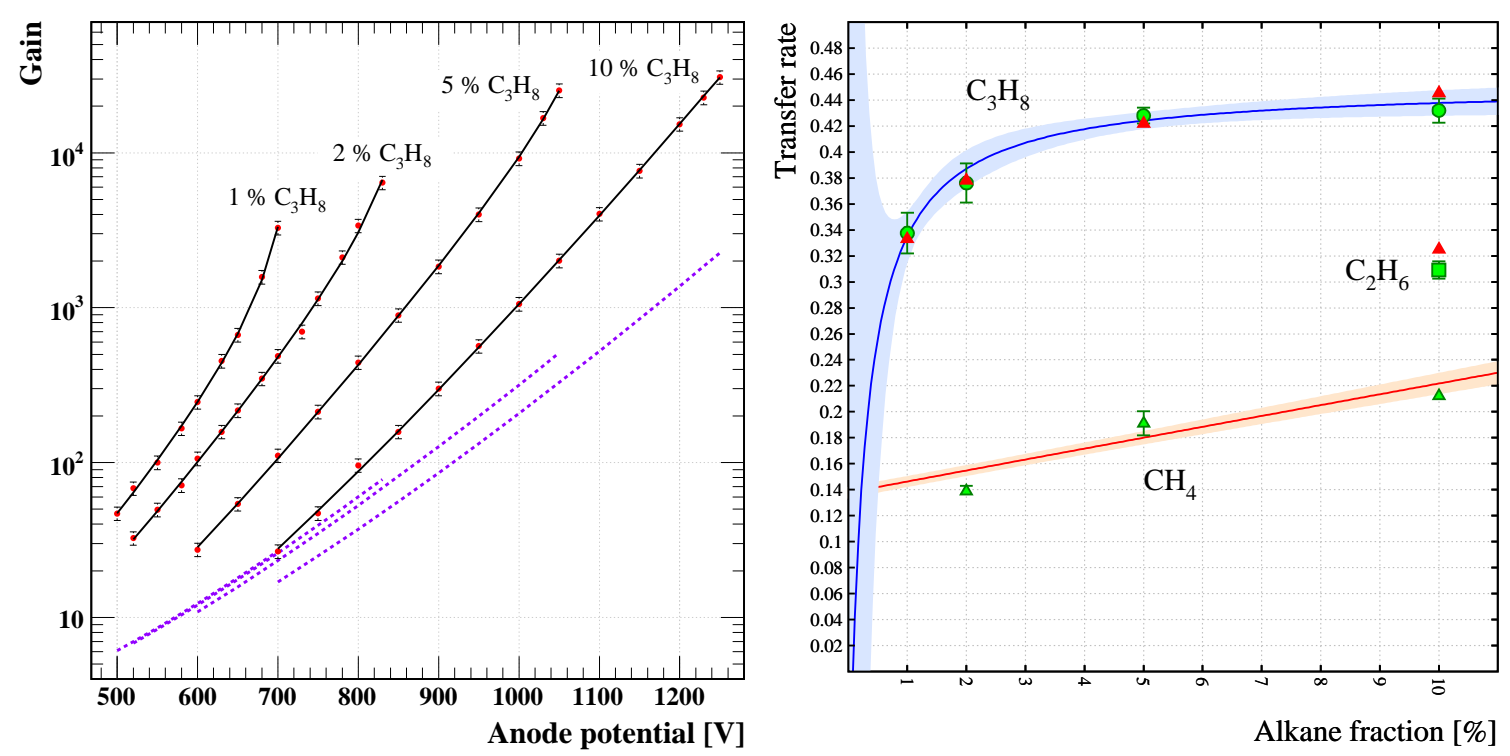

Figure 14. Left: Measured gain curves for $\mathrm{Ar}-\mathrm{C}_{3} \mathrm{H}_{8}$ mixtures from P.C. Agrawal et al. [3] (red dots) with fits of the transfer rates (black lines). For comparison, dashed purple lines show the calculated gain curves without transfer. Right: Transfer rates for argon with $10 \% \mathrm{C}_{3} \mathrm{H}_{8}$ at $p_{\text {gas }}=1$ atm fitted with $\left(a_{1} c+a_{3}\right) /(c+$ $a_{2}$ ), blue curve. Red triangles: gain scaling fixed at the weighted average. Data for other linear alkanes at the same pressure is shown for comparison. The $\mathrm{CH}_{4}$ curve is eq. (4.12) for $p=1$ with the parameters of the fit shown in figure 12. This parametrisation is not valid at small $\mathrm{CH}_{4}$ fractions because of the underlying assumption of a nearly constant photo-ionisation efficiency.

spheric pressure approximately $1 \%$ to the transfer probability. This is compatible with the upper limit of 3.6\% derived by E. Eggarter [31]. Revisiting this estimate, taking more recent cross sections into account, S.F. Biagi [15] finds an upper limit of 1.5-2\%, which is still compatible. The contribution is small because of the high energy threshold.

A scenario emerges in which energy transfer in $\mathrm{Ar}^{*}-\mathrm{CH}_{4}$ collisions is the dominant Penning process at high pressure and high $\mathrm{CH}_{4}$ concentrations. Ar*-Ar homonuclear associative ionisation surprisingly plays a role at high pressure. Photo-ionisation is the only available process at low pressures. The process also contributes, albeit at a lower level, at high pressures and high $\mathrm{CH}_{4}$ concentrations. There is no evidence for loss of excited argon atoms through processes quadratic in pressure and argon concentration, like excimer formation. These would lead to a drop of the transfer probability at higher pressure and such a drop is not observed.

The time dependence of excitation transfer is entirely determined by the model parameters of eq. (4.12), the lifetimes of the excited states and the time between collisions (figure 2).

\subsection{Argon-ethane, argon-propane and argon-isobutane mixtures}

P.C. Agrawal et al. [1-3] have published gain curves for argon with a range of admixtures, including the alkanes $\mathrm{C}_{2} \mathrm{H}_{6}(10 \%), \mathrm{C}_{3} \mathrm{H}_{8}$ (several concentrations) and $\mathrm{iC}_{4} \mathrm{H}_{10}(10 \%)$. They used the same equipment as for their Ar-Xe data (section 4.1).

Argon excimers can not ionise these admixtures, except $\mathrm{C}_{3} \mathrm{H}_{8}$ and $\mathrm{iC}_{4} \mathrm{H}_{10}$ at low pressure when the first continuum at $11.3 \mathrm{eV}$ is not suppressed (section 2.4). Since the photo-absorption 
Table 2. Transfer probabilities in argon with $10 \%$ alkane at $1 \mathrm{~atm}$.

\begin{tabular}{|l|r|r|r|}
\hline Alkane & $\begin{array}{r}\text { Ionisation potential } \\
{[\mathrm{eV}]}\end{array}$ & $\begin{array}{r}\text { Molecular weight } \\
{[\mathrm{g} / \mathrm{mol}]}\end{array}$ & Transfer probability \\
\hline $\mathrm{CH}_{4}$ & 12.65 & 16.04 & $0.212 \pm 0.002$ \\
$\mathrm{C}_{2} \mathrm{H}_{6}$ & 11.52 & 30.07 & $0.31 \pm 0.01$ \\
$\mathrm{C}_{3} \mathrm{H}_{8}$ & 10.95 & 44.096 & $0.43 \pm 0.01$ \\
$\mathrm{iC}_{4} \mathrm{H}_{1} 0$ & 10.67 & 58.123 & $0.40 \pm 0.01$ \\
\hline
\end{tabular}

cross sections (section 2.6) are substantially higher than in methane, the mean free path of photons from radiative decay is even smaller. Photon feedback is detectable, though, for low admixture concentrations (figure 14). A common gain scaling of $g=0.31$ is compatible with all $\mathrm{C}_{3} \mathrm{H}_{8}$ gain curves. No gain scaling has been imposed for the other admixtures.

A notable difference with $\mathrm{CH}_{4}$ is that the ionisation potentials $\left(11.52 \mathrm{eV}\right.$ for $\mathrm{C}_{2} \mathrm{H}_{6}, 10.95 \mathrm{eV}$ for $\mathrm{C}_{3} \mathrm{H}_{8}$ and $10.67 \mathrm{eV}$ for $\left.\mathrm{iC}_{4} \mathrm{H}_{10}\right)$ are located below the argon $3 p^{5} 4 s$ excitation levels. This results in a larger pool of excited argon atoms which can potentially transfer their energy. Indeed, comparing the $1 \mathrm{~atm}$ gain curves of argon mixed with $10 \% \mathrm{CH}_{4}$ (figure 11) and with $10 \%$ heavier alkanes (figure 14), the gain is noticeably more enhanced with the heavier alkanes.

As seen for the $\mathrm{Ar}-\mathrm{C}_{2} \mathrm{H}_{2}$ mixtures (section 4.3), the $3 p^{5} 4 s$ states have a long effective lifetime because of radiation trapping. This results in a reduced concentration dependence of their transfer probability. Since $\mathrm{C}_{3} \mathrm{H}_{8}$ is more efficient than $\mathrm{C}_{2} \mathrm{H}_{2}$ in limiting photon feedback, the $\mathrm{C}_{3} \mathrm{H}_{8}$ data has, for equal gain and equal admixture percentages, been taken at $\mathrm{a} \approx 30 \%$ higher field. The role of the $3 p^{5} 4 s$ states is therefore less prominent than in $\mathrm{Ar}-\mathrm{C}_{2} \mathrm{H}_{2}$ while the $3 p^{5} 4 p, 3 p^{5} 3 d$ and higher states should show a concentration dependence similar to that of e.g. $\mathrm{Ar}-\mathrm{CH}_{4}$.

The $\mathrm{C}_{3} \mathrm{H}_{8}$ data does not extend to sufficiently low quencher concentrations (or pressures) to give accurate information on photo-ionisation and homonuclear associative ionisation via the ratio $a_{3} / a_{2}=-0.1 \pm 2.2$, see eq. (4.7) and figure 14. Inspection of the $\chi^{2}$ curve reveals an asymmetric error bar of which the positive side can be used to derive the upper limit $a_{2}<0.012$. Combined with the collision time for this mixture $\tau_{A^{*} B}=130 \mathrm{ps}$ (table 1) and assuming full collision transfer efficiency, the lower limit on the average lifetime of the excited states is:

$$
\frac{1}{a_{2}} \frac{\tau_{A^{*} B}}{\tau_{A^{*}}}=f_{B^{+}}+f_{\bar{B}}<1, \quad \tau_{A^{*}}>11 \mathrm{~ns}(84 \% \mathrm{CL})
$$

This is indeed larger than the natural lifetime of non-metastable $3 p^{5} 4 s$ and compatible with $3 p^{5} 4 p$. We note that $f_{B^{+}}+f_{\bar{B}}$ is likely to be smaller than unity.

The transfer probabilities at $1 \mathrm{~atm}$ in argon mixed with $10 \%$ alkane, increase with molecular weight, at least for the linear alkanes (table 2). Isobutane probably falls out of this trend because it is compact and hence has a smaller collision time. At this pressure, as in the case of $\mathrm{CH}_{4}$, radiative and heteronuclear associative ionisation are dominant and of comparable importance (figure 13). With reference to eq. (1.13), two arguments can be put forward to explain the observed trend. First, the UV absorption cross section of alkanes, and therefore $f_{\text {rad }}$, increases with the molecular weight (figure 5). Second, it may be that $f_{B^{+}} /\left(f_{B^{+}}+f_{\bar{B}}\right)$ increases with the molecular weight. No 
measurements of the ionisation branching fraction have been found for these gases to support or contradict this hypothesis.

\section{Conclusion}

Gas gain measurements for Penning mixtures are notoriously hard to reconcile with Townsend coefficients. In the mixtures and at the pressures that we have investigated, they can however be understood in terms of established molecular processes.

In this report, we use the variation of gain curves with gas pressure and admixture concentration to identify the mechanisms that contribute to gas multiplication. The main processes, other than direct ionisation, responsible for the gas gain are found to be photo-ionisation and energy transfer in collisions between excited argon atoms and admixture molecules. There are hints of homonuclear associative ionisation - these are to be confirmed. Loss of excited states through excimer formation is not observed. The relative importance of these processes can be described with a simple model of which the main ingredients are:

- excitation and ionisation frequencies, which can be calculated by the Magboltz program;

- photo-absorption and -ionisation cross sections, which have been measured for many gases;

- lifetimes of excited states, which are known for most states and which can be strongly affected by radiation trapping;

- the time interval between collisions, which scales with the pressure and the concentrations, and which can be estimated from the molecular weights and diameters;

- probabilities $f_{A^{+}}, f_{B^{+}}, \ldots$ that excited molecules ionise ground-state molecules in collisions, probabilities which can be extracted from gain curves and which do not depend on the gas pressure and the admixture concentration.

The collisional ionisation probabilities are subject to a potential systematic bias as a result of ambiguity in the choice of molecular diameters. This introduces uncertainty in the number of molecular collisions before transfer occurs, but not in time evolution of Penning transfers, nor in the net transfer rates.

\section{Acknowledgments}

We would like to acknowledge Steve Biagi and Heinrich Schindler for their help.

\section{References}

[1] P.C. Agrawal and B.D. Ramsey, Use of propane as a quench gas in argon-filled proportional counters and comparison with other quench gases, Nucl. Instrum. Meth. A 273 (1988) 331.

[2] P.C. Agrawal and B.D. Ramsey, Penning gas mixtures for improving the energy resolution of proportional counters, IEEE Trans. Nucl. Sci. 36 (1989) 866. 
[3] P.C. Agrawal et al., Study of argon-based Penning gas mixtures for use in proportional counters, Nucl. Instrum. Meth. A 277 (1989) 557.

[4] J.A. Aguilera et al., Lifetimes of some 6p levels of neutral argon, Phys. Rev. A 45 (1992) 2753.

[5] K. Aho et al., Pressure-dependent decay of the $3 p^{5} 4 p$ configuration in argon excited by alpha-particles and protons, J. Phys. B 31 (1998) 4191.

[6] E. Albrecht et al., VUV absorbing vapours in n-perfluorocarbons, Nucl. Instrum. Meth. A 510 (2003) 262.

[7] G.D. Alkhazov, Ionization fluctuations in gases, Sov. Phys. Tech. Phys. 16 (1972) 1540.

[8] D. Kent Anderson, Lifetimes of the $\left(5 p^{5} 6 s\right)^{1} P_{1}$ and ${ }^{3} P_{1}$ States of Xenon, Phys. Rev. 137 (1965) A21.

[9] A. Andronic, S. Biagi, P. Braun-Munzinger, C. Garabatos and G. Tsiledakis, Drift velocity and gain in argon- and xenon-based mixtures, Nucl. Instrum. Meth. A 523 (2004) 302.

[10] J.W. Au et al., The valence shell photoabsorption of the linear alkanes, $\mathrm{C}_{n} \mathrm{H}_{2 n+2}(n=1-8)$ : absolute oscillator strengths $(7-220 \mathrm{eV})$, Chem. Phys. 173 (1993) 209.

[11] J. Berkowitz, Atomic and Molecular Photoabsorption, ch. 2, 5, 6, pp. 82, 189, 246, 252, 267. Academic Press (2002).

[12] C.G.P.M. Bernardo and J.A.N.F. Gomes, Cluster model DFT study of acetylene adsorption on the Cu (100) surface, J. Mol. Str. (Theochem) 629 (2003) 251.

[13] S.F. Biagi, E. Gabathuler and D. Duxbury, Experimental results from a microdot detector overcoated with a semiconducting layer, Nucl. Instrum. Meth. A 419 (1998) 438.

[14] S.F. Biagi, Monte Carlo simulation of electron drift and diffusion in counting gases under the influence of electric and magnetic fields, Nucl. Instrum. Meth. A 421 (1999) 234.

[15] S.F. Biagi, private communication, Oct. $1^{\text {st }} 2009$.

[16] M.J.G. Borge and J. Campos, Transition probabilities for lines arising from levels belonging to the $3 \mathrm{p}^{5} \mathrm{np}(n=4,5,6)$ configurations of Ar I, Physica B+C 119C (1983) 359.

[17] J.A.C. Broekaert, Analytical Atomic Spectrometry with Flames and Plasmas, ch. 5, p. 266. Wiley-VCH Verlag GmbH \& Co. KHaA (2005).

[18] I.K. Bronić and B. Grosswendt, Gas amplification and ionization coefficients in isobutane and argon-isobutane mixtures at low gas pressures, Nucl. Instrum. Meth. B 142 (1998) 219.

[19] H. Brunet et al., Spectroscopic and kinetic analysis of the VUV emissions of argon and argon-xenon mixtures. II. Energy transfer in Ar-Xe mixtures, J. Phys. B 15 (1982) 2945.

[20] J.H. Callomon et al., Landolt-Börnstein, Numerical Data and Functional Relationships in Science and Technology - Atomic and Molecular Physics - Structure Data of Free Polyatomic Molecules, vol. 7. Springer-Verlag Berlin, Heidelberg Germany, New York U.S.A. (1976).

[21] Wing-Fat Chan, Absolute optical oscillator strengths for electronic excitations of noble gas atoms and diatomic molecules. PhD thesis, UBC cIRcle 2429/2886, Department of Chemistry, University of British Columbia, Vancouver, British Columbia, Canada V6T 1 Z1 (1992), http://hdl.handle.net/2429/2886.

[22] W.F. Chan et al., Absolute optical oscillator strengths for the electronic excitation of atoms at high resolution. III. The photoabsorption of argon, krypton, and xenon, Phys. Rev. A 46 (1992) 149.

[23] W.F. Chan et al., The electronic spectrum of carbon dioxide. Discrete and continuum photoabsorption oscillator strengths (6-203eV), Chem. Phys. 178 (1993) 401. 
[24] M.W. Charles, Gas gain measurements in proportional counters, J. Phys. E 5 (1972) 95.

[25] X. Chen et al., Synthesis of zeolite NaA membranes with high permeance under microwave radiation on mesoporous-layer-modified macroporous substrates for gas separation,

J. Memb. Sc. 255 (2005) 201.

[26] M.B. Das and S. Karmakar, Radiative lifetimes of some excited states of neutral xenon, Eur. Phys. J. D 32 (2005) 285.

[27] M.B. Das et al., Lifetime Measurement of Some Excited States of Neutral neon, Phys. Scripta 71 (2005) 599.

[28] J.P. Desclaux, Relativistic Dirac-Fock expectation values for atoms with $Z=1$ to $Z=120$, Atom. Data Nucl. Data tables 12 (1973) 311.

[29] H. Deutsch et al., Calculated cross sections for the electron-impact ionization of excited argon atoms using the DM formalism, Int. J. Mass Spectrom. 233 (2004) 39.

[30] M.J. Druyvesteyn and F.M. Penning, The Mechanism of Electrical Discharges in Gases of Low Pressure, Rev. Mod. Phys. 12 (1940) 87 [Erratum ibid. 13 (1941) 72].

[31] E. Eggarter, Comprehensive optical and collision data for radiation action. II. Ar, J. Chem. Phys. 62 (1975) 833.

[32] P. Erman and S. Huldt, Trapping of Non-Resonance Radiation in Atoms and its Influence on Measured Lifetimes of the 4p and 5p Levels in Ar I, Physica Scripta 17 (1978) 473.

[33] P.F. Gruzdev and A.V. Loginov, Radiation lifetimes of levels of the argon atom, Opt. Spektrosk. 38 (1975) 411.

[34] J. Gulín-González et al., A molecular dynamics study of the diffusion of a hydrocarbon in $\mathrm{AlPO}_{4-5}$ molecular sieve, Revista CENIC Ciencias Químicas 34 (2003) 29.

[35] Y. Hatano, Interaction of photons with molecules-cross-sections for photoabsorption, photoionization, and photodissociation, Rad. Env. Biophys. 38 (1999) 239.

[36] A.E.D. Heylen, Maximization of argon-hydrocarbon Penning mixtures, J. Phys. D 3 (1970) 789.

[37] T. Holstein, Imprisonment of Resonance Radiation in Gases, Phys. Rev. 72 (1947) 1212.

[38] T. Holstein, Imprisonment of Resonance Radiation in Gases. II, Phys. Rev. 83 (1951) 1159.

[39] H. Horiguchi et al., Radiative lifetimes and two-body collisional deactivation rate constants in Ar for $\mathrm{Xe}\left(5 \mathrm{p}^{5} 6 \mathrm{p}\right), \mathrm{Xe}\left(5 \mathrm{p}^{5} 6 \mathrm{p}\right)$, and Xe(5p5 $\left.7 \mathrm{p}\right)$ states, J. Chem. Phys. 75 (1981) 1207.

[40] J.A. Hornbeck and J.P. Molnar, Mass Spectrometric Studies of Molecular Ions in the Noble Gases, Phys. Rev. 84 (1951) 621.

[41] H. Hotop and A. Niehaus, Reactions of Excited Atoms and Molecules with Atoms and Molecules II. Energy Analysis of Penning Electrons, Z. Phys. 228 (1969) 68.

[42] Z. Hu and E.F. Vansant, Carbon molecular sieves produced from walnut shell, Carbon 33 (1995) 561.

[43] R.E. Huffman and D.H. Katayama, Photoionization Study of Diatomic-Ion Formation in Argon, Krypton, and Xenon, J. Chem. Phys. 45 (1966) 138.

[44] T. Iijima, Molecular Structure of Propane, Bull. Chem. Soc. Japan 45 (1972) 1291.

[45] T. Iijima, Molecular Structure of Ethane. Comparison of the Structure Parameters of $\mathrm{CH}_{3} \mathrm{CD}_{3}$ in the Torsionally Excited State and in the Ground State, Bull. Chem. Soc. Japan 46 (1973) 2311. 
[46] W.P. Jesse and J. Sadauskis, Alpha-Particle Ionization in Mixtures of the Noble Gases, Phys. Rev. 88 (1952) 417.

[47] M.T. Jones et al., Branching Fractions for Penning Ionization in Quenching of $\mathrm{He}\left(2^{3} S\right), \operatorname{Ar}\left({ }^{3} P_{2,0}\right)$, and $\mathrm{Ne}\left({ }^{3} P_{2,0}\right)$ Atoms, J. Phys. Chem. 89 (1985) 4501.

[48] K. Kameta et al., Photoabsorption, photoionization, and neutral-dissociation cross sections of simple hydrocarbons in the vacuum ultraviolet range, J. Electron Spectros. Relat. Phenom. 123 (2002) 225.

[49] K. Katsuura, Ionization of Atoms by Collision with Excited Atoms, J. Chem. Phys. 42 (1965) 3771.

[50] S. Kishimoto and Y. Isozumi, On the Partial Liquefaction of Gas Mixture in a Proportional Counter Cooled at Low Temperatures, Bull. Inst. Chem. Res. 64 (1986) 25.

[51] J.Z. Klose, Experimental Lifetimes of the 5p Levels in Ar I, J. Opt. Soc. Am. 58 (1968) 1509.

[52] T.Z. Kowalski and A.R. Stopczynski, The Gas gain process in $\mathrm{Ar} / \mathrm{CO}_{2}$ filled proportional tubes, Nucl. Instrum. Meth. A 323 (1992) 289.

[53] C.T. Kuo et al., The photoabsorption spectrum of $\mathrm{CO}_{2}$ at $104-170 \mathrm{~nm}$, Chin. J. Phys. 42 (2004) 65.

[54] H. Langhoff, The origin of the third continua emitted by excited rare gases, Opt. Comm. 68 (1988) 31.

[55] P. Laporte, V. Saile, R. Reininger, U. Asaf and I.T. Steinberger, Photoionization of Xenon below the atomic ionization potential, Phys. Rev. A 28 (1983) 3613.

[56] G.M. Lawrence, Radiance Lifetimes in the Resonance Series of Ar I, Phys. Rev. 175 (1968) 40.

[57] W. Legler, Zur Statistik der Elektronenlawinen, Z. Phys. A 140 (1955) 221.

[58] W. Legler, Statistische Probleme bei Nachlieferungsprozessen und beim Townsend-Aufbau von Gasentladungen, Ann. Phys. 453 (1956) 374.

[59] E.P. De Lima et al., Fano factors of rare gases and their mixtures, Nucl. Instrum. Meth. 192 (1982) 575.

[60] R.H. Lipson et al., Recombination studies of $\mathrm{Xe}_{2}^{+}$following associative ionization of laser-excited Xe, J. Phys. Conf. Ser. 4 (2005) 216.

[61] B.A. Lombos et al., The electronic spectra of n-alkanes, J. Mol. Spectr. 24 (1967) 253.

[62] B.A. Lombos et al., The electronic spectra of normal paraffin hydrocarbons, Chem. Phys. Lett. 1 (1967) 42.

[63] B.A. Lombos et al., The far-ultraviolet spectra of branched chain paraffins, Chem. Phys. Lett. 1 (1967) 221.

[64] R.K. Manchanda et al., Ultra-high-pressure proportional counter: Part I: Argon, Nucl. Instrum. Meth. A 292 (1990) 373.

[65] F.M. Matsunaga et al., Photoionization yield and absorption coefficient of xenon in the region of 860 - 1022 Å, J. Quant. Spectr. Radiat. Trans. 5 (1965) 329.

[66] P. Millet et al., Spectroscopic and kinetic analysis of the VUV emissions of argon and argon-xenon mixtures. I. Study of pure argon, J. Phys. B 15 (1982) 2935.

[67] A. Monteil et al., Relaxation of the $\mathrm{Ne}\left(2 p_{1}\right)$ level and populations of $\mathrm{Ne}\left(1 s_{2}\right)$ and $\mathrm{Ne}\left(1 s_{4}\right)$ in an afterglow, J. Quant. Spectr. Radiat. Trans. 18 (1977) 573.

[68] J.L. Morack and C.E. Fairchild, Measured Lifetime of the $\left(3 p^{5} 4 s\right)^{3} P_{1}$ State of Argon, Phys. Rev. 163 (1967) 125. 
[69] M.S.B. Munson et al., A mass spectrometric study of homonuclear and heteronuclear rare gas molecule ions, J. Phys. Chem. 67 (1963) 1542.

[70] NIST, Energy Levels of Neutral Argon (Ar I), http://physics.nist.gov/PhysRefData/Handbook/Tables/argontable5.htm.

[71] G. Norlén, Wavelengths and Energy Levels of Ar I and Ar II based on New Interferometric Measurements in the Region 3400 - 9800 Å, Phys. Scripta 8 (1973) 249.

[72] M. Ohwa et al., Excitation mechanisms of the electron-beam-pumped atomic xenon $(5 d \rightarrow 6 p)$ laser in Ar/Xe mixtures, J. Appl. Phys. 66 (1989) 5131.

[73] I. Okazaki et al., Nanotechnological method to control the molecular weight cut-off and/or pore diameter of organic-inorganic composite membrane, J. Membr. Sc. 141 (1998) 65.

[74] F.M. Penning, Über den Einfluß sehr geringer Beimischungen auf die Zündspannung der Edelgase, Z. Phys. 46 (1928) 335.

[75] F.M. Penning, The starting potential of the glow discharge in neon argon mixtures between large parallel plates: II. Discussion of the ionisation and excitation by electrons and metastable atoms, Physica 1 (1934) 1028.

[76] G.M. Petrov et al., Electron kinetics of the e-beam pumped Ar-Xe laser, J. Phys. D 40 (2007) 4532.

[77] B.D. Ramsey and P.C. Agrawal, Quench gases for xenon-(and krypton-) filled proportional counters, Nucl. Instrum. Meth. A 273 (1988) 326.

[78] E.B. Saloman, Energy Levels and Observed Spectral Lines of Xenon, Xe I through Xe LIV, J. Phys. Chem. Ref. Data 33 (2004) 765.

[79] A. Schmitt and H. Schmoranzer, Radiative lifetimes of the $5 p^{5} 6 p$-fine-structure levels of xenon measured by beam-gas-laser spectroscopy, Phys. Lett. A 263 (1999) 193.

[80] J.P. Sephton et al., The quenching of Penning mixtures in a cylindrical proportional counter, Nucl. Instrum. Meth. A 256 (1987) 561.

[81] D.W. Setser, Quenching reactions of electronically excited atoms, in APP Spring Meeting, Diagnostics of Non-Equilibrium High Pressure Plasmas, Physikzentrum, Bad Honnef (2001), http://www.ilp.physik.uni-essen.de/doebele/Spring2001/pdf/141.pdf.

[82] D.A. Shaw et al., A study of the absolute photoabsorption, photoionisation and photodissociation cross sections and the photoionisation quantum efficiency of carbon dioxide from the ionisation threshold to $345 \AA$ A, Chem. Phys. 198 (1995) 381.

[83] B.M. Smirnov and O.B. Firsov, Ionization of an atom colliding with an excited atom, JETP Prisma 2 (1965) 478 .

[84] A.G. Stepanov et al., Dynamics of Isobutane inside Zeolite ZSM-5. A Study with Deuterium Solid-State NMR, J. Phys. Chem. B 106 (2002) 10114.

[85] F. Tokanai et al., The energy resolution of proportional counters with thin anode diameters down to 5 $\mu \mathrm{m}$, IEEE Trans. Nucl. Sci. 41 (1994) 1042.

[86] G.A. Tsongas and S.P. Koutsoyannis, A calculation of repulsive force constants of the Lennard-Jones potential for some excited states of noble gases, J. Phys. B 2 (1969) 437.

[87] N. Wainfan et al., Photoionization Efficiencies and Cross sections in $\mathrm{O}_{2}, \mathrm{~N}_{2}, \mathrm{CO}_{2}, \mathrm{~A}, \mathrm{H}_{2} \mathrm{O}, \mathrm{H}_{2}$, and $\mathrm{CH}_{4}$, Phys. Rev. 99 (1955) 542. 
[88] A.H. Walenta and T. Çonka Nurdan, Drift Chamber Experiment, in CP674, Instrumentation in Elementary Particle Physics: First ICFA Instrumentation School at the ICFA Instrumentation Center in Morelia, L. Villaseñor and V. Villanueva eds., American Institute of Physics (2003) pp. 246-265.

[89] M. Walhout et al., Lifetime of the metastable $6 \mathrm{~s}^{\prime}[1 / 2]_{0}$ clock state in xenon, Opt. Lett. 20 (1995) 1192.

[90] W. Wieme and J. Lenaerts, Excimer formation in argon, krypton, and xenon discharge afterglows between 200 and 400 K, J. Chem. Phys. 74 (1981) 483.

[91] W.L. Wiese et al., Unified set of atomic transition probabilities for neutral argon, Phys. Rev. A 39 (1989) 2461.

[92] J. Wieser et al., Vacuum ultraviolet rare gas excimer light source, Rev. Sci. Instrum. 68 (1997) 1360.

[93] M. Winter, WebElements: the periodic table on the web, http://www.webelements.com.

[94] Z. Ye et al., Gas amplification in high pressure proportional counters, Nucl. Instrum. Meth. A 329 (1993) 140.

[95] W. Zhu et al., Direct current planar excimer source, J. Phys. D 40 (2007) 3896.

[96] W. Zhu et al., Adsorption on Kureha Activated Carbon: Isotherms and Kinetics, Adsorption 11 (2005) 637. 